\title{
Exact Traveling Wave Solutions of Certain Nonlinear Partial Differential Equations Using the $\left(G^{\prime} / G^{2}\right)$-Expansion Method
}

\author{
Sekson Sirisubtawee $\mathbb{D}^{1,2}$ and Sanoe Koonprasert $\mathbb{D}^{1,2}$ \\ ${ }^{1}$ Department of Mathematics, Faculty of Applied Science, King Mongkut's University of Technology North Bangkok, \\ Bangkok 10800, Thailand \\ ${ }^{2}$ Centre of Excellence in Mathematics, CHE, Si Ayutthaya Road, Bangkok 10400, Thailand
}

Correspondence should be addressed to Sekson Sirisubtawee; sekson.s@sci.kmutnb.ac.th

Received 29 January 2018; Revised 20 March 2018; Accepted 23 April 2018; Published 3 June 2018

Academic Editor: Guozhen Lu

Copyright (c) 2018 Sekson Sirisubtawee and Sanoe Koonprasert. This is an open access article distributed under the Creative Commons Attribution License, which permits unrestricted use, distribution, and reproduction in any medium, provided the original work is properly cited.

We apply the $\left(G^{\prime} / G^{2}\right)$-expansion method to construct exact solutions of three interesting problems in physics and nanobiosciences which are modeled by nonlinear partial differential equations (NPDEs). The problems to which we want to obtain exact solutions consist of the Benny-Luke equation, the equation of nanoionic currents along microtubules, and the generalized Hirota-Satsuma coupled KdV system. The obtained exact solutions of the problems via using the method are categorized into three types including trigonometric solutions, exponential solutions, and rational solutions. The applications of the method are simple, efficient, and reliable by means of using a symbolically computational package. Applying the proposed method to the problems, we have some innovative exact solutions which are different from the ones obtained using other methods employed previously.

\section{Introduction}

Various phenomena such as shallow water waves and multicellular biological dynamics arising in the nonlinear physical sciences $[1,2]$, engineering [3, 4], and biology [5] can be modeled by a class of integrable nonlinear evolution equations which can be expressed in terms of nonlinear partial differential equations (NPDEs) of integer orders. Consequently, study of traveling wave solutions of NPDEs plays a significant role in the investigation of behaviors of nonlinear phenomena. Due to the efficiency, reliability, and easy use of symbolic software packages such as Maple or Mathematica, many powerful methods have been constructed and developed to analytically solve NPDEs with their aid. Over the last few decades, exact solutions, analytical approximate solutions, and numerical solutions of many NPDEs have been successfully obtained. The methods for obtaining exact explicit solutions of NPDEs are, for example, the $\left(G^{\prime} / G\right)$-expansion method [6-8], the $\left(G^{\prime} / G, 1 / G\right)$-expansion method [9-11], the novel $\left(G^{\prime} / G\right)$ expansion method [12], the tanh-function method [13], the exp-function method $[14,15]$, the F-expansion method [16],
Hirota's direct method [17, 18], Kudryashov method [19, 20], and the extended auxiliary equation method [21]. Examples of the methods for obtaining analytical approximate solutions to NPDEs are the variational iteration method [22, 23] (VIM), the Adomian decomposition method [24, 25] (ADM), the homotopy perturbation method $[26,27]$ (HPM), and the reduced differential transform method [28]. In addition, the examples of useful methods for solving NPDEs numerically are the generalized finite difference method [29], the finite volume method [30], the finite element method [31], the spectral collocation method [32], and the Galerkin finite element method [33]. However, we prefer, if possible, to obtain exact solutions of NPDEs.

In the recent decades, applications of the $\left(G^{\prime} / G^{2}\right)$ expansion method for solving NPDEs have been proposed in various areas of applied sciences and engineering. For example, Chen [34] gave the application of the $\left(G^{\prime} / G^{2}\right)$-expansion method for seeking exact solutions of the coupled nonlinear Klein-Gordon equation. Wen-An et al. [35] demonstrated the use of the $(w / g)$-expansion method for finding traveling wave solutions of a nonlinear evolution equation. Zayed and 
Arnous [36] investigated the use of the modified $(w / g)$ expansion method for finding traveling wave solutions of nonlinear evolution equations. The modified method can be thought as the generalization of the well-known $\left(G^{\prime} / G\right)$ expansion method introduced in [37] with the special functions $w$ and $g$ including the case of $w=G^{\prime}$ and $g=G^{2}$. In particular, Wen-An et al. [35] applied the modified method, i.e., the $\left(G^{\prime} / G^{2}\right)$-expansion method, to find the traveling wave solutions of the Vakhnenko equation. Zhouzheng [38] applied the $\left(G^{\prime} / G^{2}\right)$-expansion method to obtain the exact solutions of the modified Benjamin-Bona-Mahony (MBBM) and Ostrovsky-Benjamin-Bona-Mahony (OBBM) equations. They found that the explicit exact solutions of the equations obtained by the method are in terms of some trigonometric, hyperbolic, and rational functions. Gepreel [39] employed the extended rational $\left(G^{\prime} / G^{2}\right)$-expansion method to obtain traveling wave solutions of the first equation of two integral members of nonlinear Kadomtsev-Petviashvili (KP) hierarchy equations in mathematical physics. Mohyud-Din and Bibi [40] used the $\left(G^{\prime} / G^{2}\right)$-expansion method along with the fractional complex transform to analytically solve the space-time fractional Zakharov-Kuznetsov-Benjamin-BonaMahony (ZKBBM) and the space-time fractional coupled Burgers equations for innovative exact solutions. Their exact solutions include trigonometric, hyperbolic, and rational function solutions, while Zhang et al. [41] proposed the use of the $\left(G^{\prime} / G^{2}\right)$-expansion method for solving the Schrödinger equation with third-order dispersion.

The rest of this article is organized as follows. In Section 2, the brief description of the $\left(G^{\prime} / G^{2}\right)$-expansion method is given. In Section 3, we apply the method to some real world problems modeled by NPDEs in order to obtain their exact solutions. Finally, the conclusions are drawn in Section 4.

\section{Algorithm of the $\left(G^{\prime} / G^{2}\right)$-Expansion Method}

In this section, we provide the description of the $\left(G^{\prime} / G^{2}\right)$ expansion method which is discussed in $[34,40]$. Consider a nonlinear evolution partial differential equation (NEPDE) in two independent variables $x$ and $t$ as follows:

$$
P\left(u, u_{t}, u_{x}, u_{t t}, u_{x x}, u_{x t}, \ldots\right)=0
$$

where $u=u(x, t)$ is an unknown function of independent variables $x, t$ and $P$ is a polynomial of $u$ and its various partial derivatives in which the highest order derivatives and nonlinear terms are involved.

The main steps of the method to obtain exact solutions of NPDEs can be given as follows [42, 43].

Step 1. Convert a nonlinear partial differential equation in (1) into an ordinary differential equation (ODE) using the traveling wave transformation in a variable $\xi$ as follows:

$$
\begin{aligned}
u(x, t) & =U(\xi), \\
\xi & =k x-c t
\end{aligned}
$$

where $k$ and $c$ are nonzero arbitrary constants. It can be noted that another transform $\xi=k(x-c t)$ can be sometimes used for some certain problems. With the transformation in (2) and integrations with respect to $\xi$ as many as possible, (1) is reduced to an ODE in $U=U(\xi)$ as follows:

$$
Q\left(U, U^{\prime}, U^{\prime \prime}, U^{\prime \prime \prime}, \ldots\right)=0,
$$

where $Q$ is a polynomial of $U(\xi)$ and its various derivatives. The prime notation $\left(^{\prime}\right)$ denotes the derivative with respect to $\xi$.

Step 2. Suppose that the formal solution of the ODE in (3) can be expressed in powers of $\left(G^{\prime} / G^{2}\right)$ as follows:

$$
U(\xi)=a_{0}+\sum_{j=1}^{N}\left[a_{j}\left(\frac{G^{\prime}}{G^{2}}\right)^{j}+b_{j}\left(\frac{G^{\prime}}{G^{2}}\right)^{-j}\right],
$$

where $G=G(\xi)$ satisfies the following nonlinear ODE:

$$
\left(\frac{G^{\prime}}{G^{2}}\right)^{\prime}=\mu+\lambda\left(\frac{G^{\prime}}{G^{2}}\right)^{2},
$$

in which $\mu \neq 1$ and $\lambda \neq 0$ are integers. The unknown constants $a_{N}$ or $b_{N}$ may be zero, but both of them cannot be zero simultaneously. The coefficients $a_{0}, a_{j}, b_{j}(j=1,2, \ldots, N)$ are unknown constants to be determined at a later step.

Step 3. The value of the positive integer $N$ can be determined using the homogeneous balance principle, i.e., by balancing between the highest order derivatives and the nonlinear terms occurring in (3). More precisely, if the degree of $U(\xi)$ is $\operatorname{deg}[U(\xi)]=N$, then the degree of the other terms will be expressed as follows:

$$
\begin{aligned}
\operatorname{deg}\left[\frac{d^{q} U(\xi)}{d \xi^{q}}\right] & =N+q, \\
\operatorname{deg}\left[(U(\xi))^{p}\left(\frac{d^{q} U(\xi)}{d \xi^{q}}\right)^{s}\right] & =N p+s(N+q) .
\end{aligned}
$$

Step 4. Substituting (4) along with (5) into (3), we obtain a polynomial in $\left(G^{\prime} / G^{2}\right)$. Collecting all coefficients of likepower of $\left(G^{\prime} / G^{2}\right)^{k}(k=0, \pm 1, \pm 2, \ldots, \pm M$, where $M$ is some positive integer) and setting all of the obtained coefficients to zero, we acquire a system of nonlinear algebraic equations for the unknown constants $a_{0}, a_{j}, b_{j}(j=1,2, \ldots, N), k$, and $c$. Assume that the resulting algebraic equations can be solved for the unknown constants using symbolic software packages such as Maple.

Step 5. The general solutions of (5) can be categorized into the following three cases when $C, D$ are arbitrary nonzero constants.

If $\mu \lambda>0$, then we obtain the general solution

$$
\frac{G^{\prime}}{G^{2}}=\sqrt{\frac{\mu}{\lambda}}\left(\frac{C \cos (\sqrt{\mu \lambda} \xi)+D \sin (\sqrt{\mu \lambda} \xi)}{D \cos (\sqrt{\mu \lambda} \xi)-C \sin (\sqrt{\mu \lambda} \xi)}\right) .
$$


If $\mu \lambda<0$, then we obtain the general solution

$$
\frac{G^{\prime}}{G^{2}}=\frac{1}{2 \lambda}\left(2 \sqrt{|\mu \lambda|}-\frac{4 C \sqrt{|\mu \lambda|} e^{2 \xi \sqrt{|\mu \lambda|}}}{C e^{2 \xi \sqrt{|\mu \lambda|}}-D}\right),
$$

which is equivalent to

$$
\begin{aligned}
& \frac{G^{\prime}}{G^{2}} \\
& =-\frac{\sqrt{|\mu \lambda|}}{\lambda}\left(\frac{C \sinh (2 \sqrt{|\mu \lambda| \xi})+C \cosh (2 \sqrt{|\mu \lambda|})+D}{C \sinh (2 \sqrt{|\mu \lambda|})+C \cosh (2 \sqrt{|\mu \lambda|})-D}\right) .
\end{aligned}
$$

If $\mu=0$ and $\lambda \neq 0$, then we obtain the general solution

$$
\frac{G^{\prime}}{G^{2}}=-\frac{C}{\lambda(C \xi+D)} .
$$

The explicit exact solutions of (1) can be obtained by inserting the values of $a_{0}, a_{j}, b_{j}(j=1,2, \ldots, N), k, c$ and the solutions in (7)-(10) into (4) with the transformation in (2).

\section{Applications of the $\left(G^{\prime} / G^{2}\right)$-Expansion Method}

In this section, we will demonstrate the use of the $\left(G^{\prime} / G^{2}\right)$ expansion method on three of the interesting problems in mathematical physics.

3.1. The Benney-Luke Equation. In this section, we will provide a use of the $\left(G^{\prime} / G^{2}\right)$-expansion method for seeking exact solitary wave solutions of the Benney-Luke equation, which is used to approximate the full water wave equations and appropriately described two-way water wave propagation with surface tension. The equation can be written in the following form $[44,45]$ :

$$
u_{t t}-u_{x x}+\alpha u_{x x x x}-\beta u_{x x t t}+u_{t} u_{x x}+2 u_{x} u_{x t}=0,
$$

where $\alpha, \beta$ are the positive integers such that their difference is in terms of the inverse bond number capturing the effects of gravity forces and surface tension.

Using the traveling wave transformation $\xi=k x-c t$, (11) is converted into the following ODE in the variable $U=U(\xi)$ :

$$
\left(c^{2}-k^{2}\right) U^{\prime \prime}+\left(\alpha k^{4}-\beta k^{2} c^{2}\right) U^{(4)}-3 c k^{2} U^{\prime} U^{\prime \prime}=0 .
$$

Integrating (12) with respect to $\xi$ once and then choosing the constant of integration to be zero, we obtain the following ODE:

$$
\begin{aligned}
& 2\left(c^{2}-k^{2}\right) U^{\prime}+2\left(\alpha k^{4}-\beta k^{2} c^{2}\right) U^{\prime \prime \prime}-3 c k^{2}\left(U^{\prime}\right)^{2} \\
& \quad=0
\end{aligned}
$$

for which the homogeneous balance principle is applied. Following Step 3 of the mentioned method, the highest order derivative $U^{\prime \prime \prime}$ and the nonlinear term of the highest order $\left(U^{\prime}\right)^{2}$ are balanced via using formula (6) as follows:

$$
\operatorname{deg}\left[U^{\prime \prime \prime}\right]=N+3=\operatorname{deg}\left[\left(U^{\prime}\right)^{2}\right]=2(N+1),
$$

which leads to $N=1$. Hence, the form of exact solutions of the ODE in (13) using the $\left(G^{\prime} / G^{2}\right)$-expansion method can be expressed as

$$
U(\xi)=a_{0}+a_{1}\left(\frac{G^{\prime}}{G^{2}}\right)+b_{1}\left(\frac{G^{\prime}}{G^{2}}\right)^{-1},
$$

where $a_{0}, a_{1}, b_{1}$ are unknown constants. Substituting (15) into (13) along with (5), then collecting all the coefficients with the same power of $\left(G^{\prime} / G^{2}\right)^{i},(i=0, \pm 1, \pm 2, \ldots)$, and finally setting these resulting coefficients to be zero, we consequently obtain the following system of algebraic equations in $a_{0}, a_{1}, b_{1}, k, c, \mu, \lambda, \alpha, \beta$ :

$$
\begin{aligned}
& \left(\frac{G^{\prime}}{G^{2}}\right)^{-4}:-12 \alpha k^{4} \mu^{3} b_{1}+12 \beta c^{2} k^{2} \mu^{3} b_{1} \\
& -3 c k^{2} \mu^{2} b_{1}^{2}=0 \\
& \left(\frac{G^{\prime}}{G^{2}}\right)^{-2}:-16 \alpha k^{4} \lambda \mu^{2} b_{1}+16 \beta c^{2} k^{2} \lambda \mu^{2} b_{1} \\
& -6 c k^{2} \lambda \mu b_{1}^{2}+6 c k^{2} \mu^{2} a_{1} b_{1}-2 c^{2} \mu b_{1}+2 k^{2} \mu b_{1}=0 \\
& \left(\frac{G^{\prime}}{G^{2}}\right)^{0}:-4 \alpha k^{4} \lambda^{2} \mu b_{1}+4 \alpha k^{4} \lambda \mu^{2} a_{1}+4 \beta c^{2} k^{2} \lambda^{2} \mu b_{1} \\
& -4 \beta c^{2} k^{2} \lambda \mu^{2} a_{1}-3 c k^{2} \lambda^{2} b_{1}^{2}+12 c k^{2} \lambda \mu a_{1} b_{1} \\
& -3 c k^{2} \mu^{2} a_{1}^{2}-2 c^{2} \lambda b_{1}+2 c^{2} \mu a_{1}+2 k^{2} \lambda b_{1} \\
& -2 k^{2} \mu a_{1}=0, \\
& \left(\frac{G^{\prime}}{G^{2}}\right)^{2}: 16 \alpha k^{4} \lambda^{2} \mu a_{1}-16 \beta c^{2} k^{2} \lambda^{2} \mu a_{1}+6 c k^{2} \lambda^{2} a_{1} b_{1} \\
& -6 c k^{2} \lambda \mu a_{1}^{2}+2 c^{2} \lambda a_{1}-2 k^{2} \lambda a_{1}=0 \\
& \left(\frac{G^{\prime}}{G^{2}}\right)^{4}: 12 \alpha k^{4} \lambda^{3} a_{1}-12 \beta c^{2} k^{2} \lambda^{3} a_{1}-3 c k^{2} \lambda^{2} a_{1}^{2}=0
\end{aligned}
$$

Solving the obtained algebraic system (16) by use of Maple, we get the following three cases.

Case 1.

$$
\begin{aligned}
& a_{0}=a_{0}, \\
& a_{1}=0, \\
& b_{1}=\mp \frac{4 k(\alpha-\beta) \mu}{\sqrt{4 \alpha k^{2} \lambda \mu+1} \sqrt{4 \beta k^{2} \lambda \mu+1}} \\
& k=k \\
& c= \pm \frac{\sqrt{4 \alpha k^{2} \lambda \mu+1} k}{\sqrt{4 \beta k^{2} \lambda \mu+1}},
\end{aligned}
$$

where $a_{0}, k, \alpha, \beta, \mu, \lambda$ are arbitrary constants. 
Case 2.

$$
\begin{aligned}
& a_{0}=a_{0}, \\
& a_{1}= \pm \frac{4 k(\alpha-\beta) \lambda}{\sqrt{4 \alpha k^{2} \lambda \mu+1} \sqrt{4 \beta k^{2} \lambda \mu+1}}, \\
& b_{1}=0, \\
& k=k, \\
& c= \pm \frac{\sqrt{4 \alpha k^{2} \lambda \mu+1} k}{\sqrt{4 \beta k^{2} \lambda \mu+1}},
\end{aligned}
$$

where $a_{0}, k, \alpha, \beta, \mu, \lambda$ are arbitrary constants.

Case 3.

$$
\begin{aligned}
& a_{0}=a_{0}, \\
& a_{1}= \pm \frac{4 k(\alpha-\beta) \lambda}{\sqrt{16 \alpha k^{2} \lambda \mu+1} \sqrt{16 \beta k^{2} \lambda \mu+1}},
\end{aligned}
$$

$$
\begin{aligned}
& b_{1}=\mp \frac{4 k(\alpha-\beta) \mu}{\sqrt{16 \alpha k^{2} \lambda \mu+1} \sqrt{16 \beta k^{2} \lambda \mu+1}}, \\
& k=k, \\
& c= \pm \frac{\sqrt{16 \alpha k^{2} \lambda \mu+1} k}{\sqrt{16 \beta k^{2} \lambda \mu+1}},
\end{aligned}
$$

where $a_{0}, k, \alpha, \beta, \mu, \lambda$ are arbitrary constants.

When we substitute the above three cases of the obtained parameters along with the functions $G^{\prime} / G^{2}$ specified in (7)(10) into the solution form (15), we can write three results of solutions of (11) as follows.

Result 1. From Case 1 in (17), we have $\xi=k x \mp$ $\left(\sqrt{4 \alpha k^{2} \lambda \mu+1} k / \sqrt{4 \beta k^{2} \lambda \mu+1}\right) t$ and the following exact solutions.

When $\mu \lambda>0$, the trigonometric function solution corresponding to the parameter values in Case 1 can be written as

$$
u_{1}^{1}(x, t)=a_{0} \mp \frac{4 k(\alpha-\beta) \sqrt{\mu \lambda}}{\sqrt{4 \alpha k^{2} \lambda \mu+1} \sqrt{4 \beta k^{2} \lambda \mu+1}}\left(\frac{C \cos (\sqrt{\mu \lambda} \xi)+D \sin (\sqrt{\mu \lambda} \xi)}{D \cos (\sqrt{\mu \lambda} \xi)-C \sin (\sqrt{\mu \lambda} \xi)}\right)^{-1} .
$$

When $\mu \lambda<0$, the exponential function solution associated with the parameter values in Case 1 can be expressed as

$$
\begin{aligned}
& u_{2}^{1}(x, t)=a_{0} \mp \frac{8 k(\alpha-\beta) \mu \lambda}{\sqrt{4 \alpha k^{2} \lambda \mu+1} \sqrt{4 \beta k^{2} \lambda \mu+1}}(2 \sqrt{|\mu \lambda|} \\
& \left.-\frac{4 C \sqrt{|\mu \lambda|} e^{2 \xi \sqrt{|\mu \lambda|}}}{C e^{2 \xi \sqrt{|\mu \lambda|}}-D}\right)^{-1} .
\end{aligned}
$$

$$
u_{1}^{2}(x, t)=a_{0} \pm \frac{4 k(\alpha-\beta) \sqrt{\mu \lambda}}{\sqrt{4 \alpha k^{2} \lambda \mu+1} \sqrt{4 \beta k^{2} \lambda \mu+1}}\left(\frac{C \cos (\sqrt{\mu \lambda} \xi)+D \sin (\sqrt{\mu \lambda} \xi)}{D \cos (\sqrt{\mu \lambda} \xi)-C \sin (\sqrt{\mu \lambda} \xi)}\right)
$$

When $\mu \lambda<0$, the exponential function solution associated with the parameter values in Case 2 can be expressed as

$$
\begin{aligned}
& u_{2}^{2}(x, t)=a_{0} \\
& \pm \frac{2 k(\alpha-\beta)}{\sqrt{4 \alpha k^{2} \lambda \mu+1} \sqrt{4 \beta k^{2} \lambda \mu+1}}(2 \sqrt{|\mu \lambda|} \\
& \left.\quad-\frac{4 C \sqrt{|\mu \lambda|} e^{2 \xi \sqrt{|\mu \lambda|}}}{C e^{2 \xi \sqrt{|\mu \lambda|}}-D}\right) .
\end{aligned}
$$

When $\mu=0, \lambda \neq 0$, the exact solution corresponding to the parameter values in Case 1 is $u_{3}^{1}(x, t)=a_{0}$, which is the constant solution.

Result 2. From Case 2 in (18), we have $\xi=k x \mp$ $\left(\sqrt{4 \alpha k^{2} \lambda \mu+1} k / \sqrt{4 \beta k^{2} \lambda \mu+1}\right) t$ and the following exact solutions.

When $\mu \lambda>0$, the trigonometric function solution corresponding to the parameter values in Case 2 can be written as
When $\mu=0, \lambda \neq 0$, the rational function solution corresponding to the parameter values in Case 2 can be expressed as

$$
u_{3}^{2}(x, t)=a_{0} \mp 4 k(\alpha-\beta)\left(\frac{C}{C \xi+D}\right)
$$

for which the traveling wave transformation for this case is $\xi=k(x \mp t)$.

Result 3. From Case 3 in (19), we have $\xi=k x \mp$ $\left(\sqrt{16 \alpha k^{2} \lambda \mu+1} k / \sqrt{16 \beta k^{2} \lambda \mu+1}\right) t$ and the following exact solutions. 
When $\mu \lambda>0$, the trigonometric function solution corresponding to the parameter values in Case 3 can be written as

$$
\begin{aligned}
u_{1}^{3}(x, t)= & a_{0} \pm \frac{4 k(\alpha-\beta) \sqrt{\mu \lambda}}{\sqrt{16 \alpha k^{2} \lambda \mu+1} \sqrt{16 \beta k^{2} \lambda \mu+1}}\left(\frac{C \cos (\sqrt{\mu \lambda} \xi)+D \sin (\sqrt{\mu \lambda} \xi)}{D \cos (\sqrt{\mu \lambda} \xi)-C \sin (\sqrt{\mu \lambda} \xi)}\right) \\
\mp & \frac{4 k(\alpha-\beta) \sqrt{\mu \lambda}}{\sqrt{16 \alpha k^{2} \lambda \mu+1} \sqrt{16 \beta k^{2} \lambda \mu+1}}\left(\frac{C \cos (\sqrt{\mu \lambda} \xi)+D \sin (\sqrt{\mu \lambda} \xi)}{D \cos (\sqrt{\mu \lambda} \xi)-C \sin (\sqrt{\mu \lambda} \xi)}\right)^{-1} .
\end{aligned}
$$

When $\mu \lambda<0$, the exponential function solution associated with the parameter values in Case 3 can be expressed as

$$
\begin{aligned}
& u_{2}^{3}(x, t)=a_{0} \\
& \pm \frac{2 k(\alpha-\beta)}{\sqrt{16 \alpha k^{2} \lambda \mu+1} \sqrt{16 \beta k^{2} \lambda \mu+1}}(2 \sqrt{|\mu \lambda|} \\
& \left.-\frac{4 C \sqrt{|\mu \lambda|} e^{2 \xi \sqrt{|\mu \lambda|}}}{C e^{2 \xi \sqrt{|\mu \lambda|}}-D}\right) \\
& \mp \frac{8 k(\alpha-\beta) \mu \lambda}{\sqrt{16 \alpha k^{2} \lambda \mu+1} \sqrt{16 \beta k^{2} \lambda \mu+1}}(2 \sqrt{|\mu \lambda|} \\
& \left.-\frac{4 C \sqrt{|\mu \lambda|} e^{2 \xi \sqrt{|\mu \lambda|}}}{C e^{2 \xi \sqrt{|\mu \lambda|}}-D}\right)^{-1} .
\end{aligned}
$$

When $\mu=0, \lambda \neq 0$, the rational function solution corresponding to the parameter values in Case 3 can be expressed as

$$
u_{3}^{3}(x, t)=a_{0} \mp \frac{4 k(\alpha-\beta) C}{C \xi+D}
$$

for which the traveling wave transformation for this case is $\xi=k(x \mp t)$.

Akter and Akbar [44] utilized the modified simple equation method to obtain exact solutions of (11) which were written as fractions of exponential functions. They can be transformed into the tanh and coth functions for which the arbitrary constants are selected appropriately as shown in their paper. Islam et al. [45] demonstrated the application of the improved $F$-expansion method with Riccati equation to obtain exact traveling wave solutions of (11). Their results were expressed in terms of the following functions:

(i) tanh, coth, their reciprocals, and their summations

(ii) tan, cot, their reciprocals, and their summations

(iii) rational functions.
However, our obtained results in (20)-(27) are more generalized than the ones described above; i.e., selecting the appropriate constants $C$ and $D$ in our solutions can lead to the solutions obtained by other existing methods mentioned previously. Here we present the plots of the exact solution $u_{2}^{1}(x, t)$ in (21) with the positive formula using the following parameter values: $\alpha=2, \beta=3, k=1, a_{0}=1, \mu=0.5, \lambda=$ $-1, C=D=1$. The three-dimensional and two-dimensional plots of this solution are portrayed in Figure 1 demonstrating the solitary wave solution of kink type.

3.2. Equation of Nanoionic Currents along Microtubules. In this section, we will show an application of the $\left(G^{\prime} / G^{2}\right)$-expansion method in nanobiosciences. One of the important models in such fields is the nonlinear transmission line model for nanoionic currents along microtubules (MTs) segmented into identical elementary rings (ERs). The model is playing an important role in cellular signalling and the elaborated details of derivation of the equation can be found in $[46,47]$. The equation of nanoionic currents along MTs is described as follows [47]:

$$
\begin{aligned}
& \frac{l^{2}}{3} u_{x x x}+\frac{Z^{3 / 2}}{l}\left(w G_{0}-2 \delta C_{0}\right) u u_{t}+2 u_{x}+\frac{Z C_{0}}{l} u_{t} \\
& +\frac{1}{l}\left(R Z^{-1}-G_{0} Z\right) u=0,
\end{aligned}
$$

where $R=0.34 \times 10^{9} \Omega$ is the resistance of the ER with length $l=8 \times 10^{-9} \mathrm{~m}, C_{0}=1.8 \times 10^{-15} \mathrm{~F}$ is the total maximal capacitance of the ER, $G_{0}=1.1 \times 10^{-13} \mathrm{Si}$ is conductance of pertaining nanopores (NPs), and $Z=5.56 \times 10^{10} \Omega$ is the characteristic impedance of the system. Parameters $\omega$ and $\delta$ represent conductance of NPs in ER and nonlinearity of ER capacitor, respectively.

Using the dimensionless wave variable $\xi=(1 / l) x-(c / \tau) t$, where $c$ is the dimensionless velocity of wave and $\tau=R C_{0}=$ $0.6 \times 10^{-6} \mathrm{~s}$ is the characteristic time of charging ER capacitor, we obtain the traveling wave transformation $u(x, t)=U(\xi)$ and then (28) is converted to the following new ODE:

$$
U^{\prime \prime \prime}+\alpha c U U^{\prime}+(6-\beta c) U^{\prime}+\gamma U=0
$$

where $\alpha=\left(3 Z^{3 / 2} / \tau\right)\left(2 \delta C_{0}-w G_{0}\right), \beta=3 Z C_{0} / \tau$, and $\gamma=$ $3\left(R Z^{-1}-G_{0} Z\right)$. 


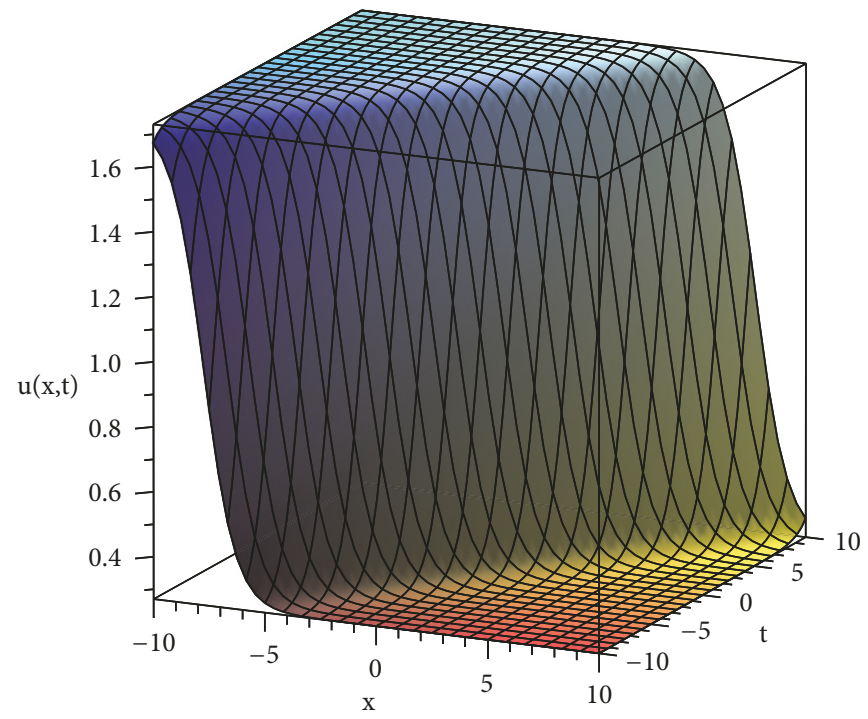

(a) 3D graph

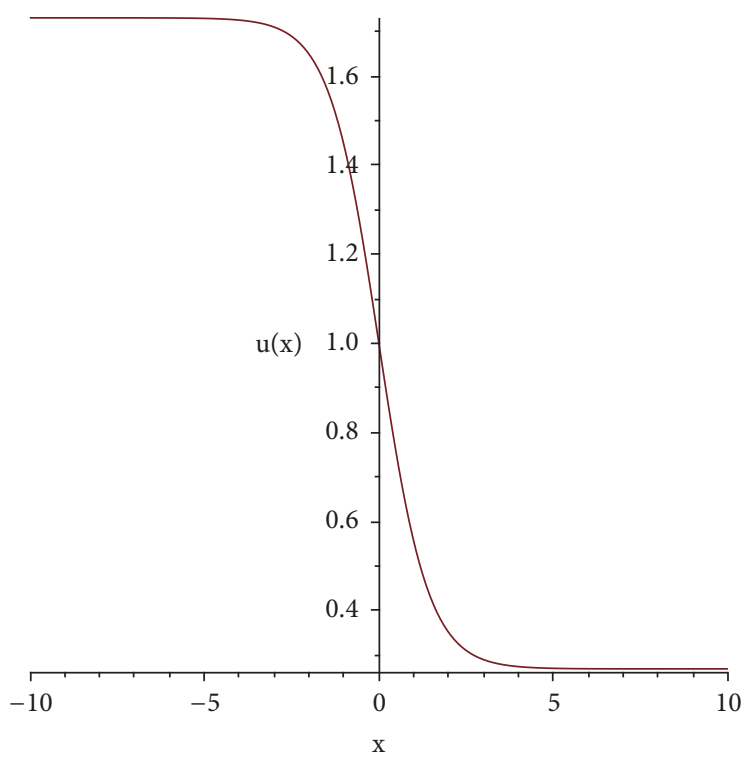

(b) 2 D graph when $t=0$

FIGURE 1: The solitary wave solutions of kink type for $u_{2}^{1}(x, t)$ in (21) when $\alpha=2, \beta=3, k=1, a_{0}=1, \mu=0.5, \lambda=-1$, and $C=D=1$.

Balancing between the highest order derivative $U^{\prime \prime \prime}$ and the nonlinear term of the highest order $U U^{\prime}$ by using formula (6), we obtain

$$
\begin{aligned}
\operatorname{deg}\left[U^{\prime \prime \prime}\right] & =N+3=\operatorname{deg}\left[U U^{\prime}\right]=2 N+1 \Longrightarrow \\
N & =2 .
\end{aligned}
$$

Thus, the form of exact solutions of (29) using the $\left(G^{\prime} / G^{2}\right)$-expansion method can be expressed as

$$
\begin{aligned}
U(\xi)= & a_{0}+a_{1}\left(\frac{G^{\prime}}{G^{2}}\right)+a_{2}\left(\frac{G^{\prime}}{G^{2}}\right)^{2}+b_{1}\left(\frac{G^{\prime}}{G^{2}}\right)^{-1} \\
& +b_{2}\left(\frac{G^{\prime}}{G^{2}}\right)^{-2}
\end{aligned}
$$

where $a_{0}, a_{1}, a_{2}, b_{1}, b_{2}$ are unknown constants. Substituting (31) into (29) along with (5), then collecting all the coefficients with the same power of $\left(G^{\prime} / G^{2}\right)^{i},(i=0, \pm 1, \pm 2, \ldots)$, and finally setting these resulting coefficients to be zero, we consequently obtain the following system of algebraic equations in $a_{0}, a_{1}, a_{2}, b_{1}, b_{2}, c, \mu, \lambda, \alpha, \beta, \gamma$ :

$$
\begin{aligned}
& \left(\frac{G^{\prime}}{G^{2}}\right)^{-5}:-2 \alpha c \mu b_{2}^{2}-24 \mu^{3} b_{2}=0 \\
& \left(\frac{G^{\prime}}{G^{2}}\right)^{-4}:-3 \alpha c \mu b_{1} b_{2}-6 \mu^{3} b_{1}=0 \\
& \left(\frac{G^{\prime}}{G^{2}}\right)^{-3}:-2 \alpha c \lambda b_{2}^{2}-2 \alpha c \mu a_{0} b_{2}-\alpha c \mu b_{1}^{2}+2 \beta c \mu b_{2}
\end{aligned}
$$

$$
\begin{aligned}
& -40 \lambda \mu^{2} b_{2}-12 \mu b_{2}=0, \\
& \left(\frac{G^{\prime}}{G^{2}}\right)^{-2}:-3 \alpha c \lambda b_{1} b_{2}-\alpha c \mu a_{0} b_{1}-\alpha c \mu a_{1} b_{2}+\beta c \mu b_{1} \\
& -8 \lambda \mu^{2} b_{1}+\gamma b_{2}-6 \mu b_{1}=0,
\end{aligned}
$$$$
\left(\frac{G^{\prime}}{G^{2}}\right)^{-1}:-2 \alpha c \lambda a_{0} b_{2}-\alpha c \lambda b_{1}^{2}+2 \beta c \lambda b_{2}-16 \lambda^{2} \mu b_{2}
$$$$
+\gamma b_{1}-12 \lambda b_{2}=0,
$$$$
\left(\frac{G^{\prime}}{G^{2}}\right)^{0}:-\alpha c \lambda a_{0} b_{1}-\alpha c \lambda a_{1} b_{2}+\alpha c \mu a_{0} a_{1}+\alpha c \mu a_{2} b_{1}
$$$$
+\beta c \lambda b_{1}-\beta c \mu a_{1}-2 \lambda^{2} \mu b_{1}+2 \lambda \mu^{2} a_{1}+\gamma a_{0}-6 \lambda b_{1}
$$$$
+6 \mu a_{1}=0 \text {, }
$$

$$
\begin{aligned}
& \left(\frac{G^{\prime}}{G^{2}}\right)^{1}: 2 \alpha c \mu a_{0} a_{2}+\alpha c \mu a_{1}^{2}-2 \beta c \mu a_{2}+16 \lambda \mu^{2} a_{2} \\
& \quad+\gamma a_{1}+12 \mu a_{2}=0,
\end{aligned}
$$$$
\left(\frac{G^{\prime}}{G^{2}}\right)^{2}: \alpha c \lambda a_{0} a_{1}+\alpha c \lambda a_{2} b_{1}+3 \alpha c \mu a_{1} a_{2}-\beta c \lambda a_{1}
$$$$
+8 \lambda^{2} \mu a_{1}+\gamma a_{2}+6 \lambda a_{1}=0,
$$$$
\left(\frac{G^{\prime}}{G^{2}}\right)^{3}: 2 \alpha c \lambda a_{0} a_{2}+\alpha c \lambda a_{1}^{2}+2 \alpha c \mu a_{2}^{2}-2 \beta c \lambda a_{2}
$$$$
+40 \lambda^{2} \mu a_{2}+12 \lambda a_{2}=0,
$$ 


$$
\begin{aligned}
& \left(\frac{G^{\prime}}{G^{2}}\right)^{4}: 3 \alpha c \lambda a_{1} a_{2}+6 \lambda^{3} a_{1}=0 \\
& \left(\frac{G^{\prime}}{G^{2}}\right)^{5}: 2 \alpha c \lambda a_{2}^{2}+24 \lambda^{3} a_{2}=0
\end{aligned}
$$

Using Maple to solve algebraic system (32), we obtain the following three cases.

Case 1.

$$
\begin{aligned}
& a_{0}=\frac{\beta c-8 \lambda \mu-6}{\alpha c}, \\
& a_{1}=0, \\
& a_{2}=0, \\
& b_{1}=0, \\
& b_{2}=-\frac{12 \mu^{2}}{\alpha c}, \\
& c=c,
\end{aligned}
$$

with $c \neq 0, \mu, \lambda$ which are arbitrary constants.

Case 2.

$$
\begin{aligned}
& a_{0}=\frac{\beta c-8 \lambda \mu-6}{\alpha c}, \\
& a_{1}=0, \\
& a_{2}=-\frac{12 \lambda^{2}}{\alpha c}, \\
& b_{1}=0, \\
& b_{2}=0, \\
& c=c,
\end{aligned}
$$

with $c \neq 0, \mu, \lambda$ which are arbitrary constants.

Case 3.

$$
\begin{aligned}
& a_{0}=\frac{\beta c-8 \lambda \mu-6}{\alpha c}, \\
& a_{1}=0, \\
& a_{2}=-\frac{12 \lambda^{2}}{\alpha c}, \\
& b_{1}=0, \\
& b_{2}=-\frac{12 \mu^{2}}{\alpha c}, \\
& c=c
\end{aligned}
$$

with $c \neq 0, \mu, \lambda$ which are arbitrary constants.
Inserting the above three cases in (33)-(35) along with the functions $G^{\prime} / G^{2}$ described in (7)-(10) into the solution form (31), we attain three results of solutions of (28) as follows.

Result 1. Using parameter values of Case 1 in (33) and $\xi=$ $(1 / l) x-(c / \tau) t$, we have the following exact solutions.

When $\mu \lambda>0$, the trigonometric function solution of (28) can be written as

$$
\begin{aligned}
u_{1}^{1}(x, t) & \\
= & \frac{\beta c-8 \mu \lambda-6}{\alpha c} \\
& -\frac{12 \mu \lambda}{\alpha c}\left(\frac{C \cos (\sqrt{\mu \lambda} \xi)+D \sin (\sqrt{\mu \lambda} \xi)}{D \cos (\sqrt{\mu \lambda} \xi)-C \sin (\sqrt{\mu \lambda} \xi)}\right)^{-2} .
\end{aligned}
$$

When $\mu \lambda<0$, the exponential function solution of (28) can be expressed as

$$
\begin{aligned}
u_{2}^{1}(x, t) & \frac{\beta c-8 \mu \lambda-6}{\alpha c} \\
& \quad-\frac{48 \mu^{2} \lambda^{2}}{\alpha c}\left(2 \sqrt{|\mu \lambda|}-\frac{4 C \sqrt{|\mu \lambda|} e^{2 \xi \sqrt{|\mu \lambda|}}}{C e^{2 \xi \sqrt{|\mu \lambda|}}-D}\right)^{-2} .
\end{aligned}
$$

When $\mu=0, \lambda \neq 0$, the exact solution of (28) is the constant solution $u_{3}^{1}(x, t)=(\beta c-6) / \alpha c$.

Result 2. Using parameter values of Case 2 in (34) and $\xi=$ $(1 / l) x-(c / \tau) t$, we have the following exact solutions.

When $\mu \lambda>0$, the trigonometric function solution of (28) can be written as

$$
\begin{aligned}
u_{1}^{2}(x, t) & \\
= & \frac{\beta c-8 \mu \lambda-6}{\alpha c} \\
& -\frac{12 \mu \lambda}{\alpha c}\left(\frac{C \cos (\sqrt{\mu \lambda} \xi)+D \sin (\sqrt{\mu \lambda} \xi)}{D \cos (\sqrt{\mu \lambda} \xi)-C \sin (\sqrt{\mu \lambda} \xi)}\right)^{2} .
\end{aligned}
$$

When $\mu \lambda<0$, the exponential function solution of (28) can be expressed as

$$
\begin{aligned}
u_{2}^{2}(x, t)= & \frac{\beta c-8 \mu \lambda-6}{\alpha c} \\
& -\frac{3}{\alpha c}\left(2 \sqrt{|\mu \lambda|}-\frac{4 C \sqrt{|\mu \lambda|} e^{2 \xi \sqrt{|\mu \lambda|}}}{C e^{2 \xi \sqrt{|\mu \lambda|}}-D}\right)^{2} .
\end{aligned}
$$

When $\mu=0, \lambda \neq 0$, the rational function solution of (28) can be expressed as

$$
u_{3}^{2}(x, t)=\frac{\beta c-6}{\alpha c}-\frac{12 C^{2}}{\alpha c(C \xi+D)^{2}} .
$$


Result 3. Using parameter values of Case 3 in (35) and $\xi=$ $(1 / l) x-(c / \tau) t$, we have the following exact solutions.

When $\mu \lambda>0$, the trigonometric function solution of (28) can be written as

$$
\begin{gathered}
u_{1}^{3}(x, t) \\
=\frac{\beta c-8 \mu \lambda-6}{\alpha c} \\
\quad-\frac{12 \mu \lambda}{\alpha c}\left(\frac{C \cos (\sqrt{\mu \lambda} \xi)+D \sin (\sqrt{\mu \lambda} \xi)}{D \cos (\sqrt{\mu \lambda} \xi)-C \sin (\sqrt{\mu \lambda} \xi)}\right)^{2} \\
\quad-\frac{12 \mu \lambda}{\alpha c}\left(\frac{C \cos (\sqrt{\mu \lambda} \xi)+D \sin (\sqrt{\mu \lambda} \xi)}{D \cos (\sqrt{\mu \lambda} \xi)-C \sin (\sqrt{\mu \lambda} \xi)}\right)^{-2} .
\end{gathered}
$$

When $\mu \lambda<0$, the exponential function solution of (28) can be expressed as

$$
\begin{aligned}
& u_{2}^{3}(x, t) \\
& =\frac{\beta c-8 \mu \lambda-6}{\alpha c} \\
& -\frac{3}{\alpha c}\left(2 \sqrt{|\mu \lambda|}-\frac{4 C \sqrt{|\mu \lambda|} e^{2 \xi \sqrt{|\mu \lambda|}}}{C e^{2 \xi \sqrt{|\mu \lambda|}}-D}\right)^{2} \\
& -\frac{48 \mu^{2} \lambda^{2}}{\alpha c}\left(2 \sqrt{|\mu \lambda|}-\frac{4 C \sqrt{|\mu \lambda|} e^{2 \xi \sqrt{|\mu \lambda|}}}{C e^{2 \xi \sqrt{|\mu \lambda|}}-D}\right)^{-2} .
\end{aligned}
$$

When $\mu=0, \lambda \neq 0$, the rational function solution of (28) can be expressed as

$$
u_{3}^{3}(x, t)=\frac{\beta c-6}{\alpha c}-\frac{12 C^{2}}{\alpha c(C \xi+D)^{2}}
$$

Satarić et al. [46] firstly proposed model (28) and obtained its analytical solution by converting (28) with the change of variables and the use of the appropriate boundary conditions into the solvable ODE in a new variable. The analytical solution was expressed in terms of a square of the exponential secant function. Later, Sekulić et al. [47] applied the modified extended tanh-function method to solve (28) for exact traveling wave solutions. The solutions were written as the square of the following functions: tan, cot, tanh, and coth. It is not difficult to verify that the mathematical structures of their exact solutions are the particular cases of those of our solutions as shown in (36), (37), (38), (39), (41), and (42). Meanwhile our rational function solutions do not appear in their work. In addition, Zayed and Alurrfi [48] utilized the $\left(G^{\prime} / G, 1 / G\right)$-expansion method to analytically obtain the exact traveling wave solutions of problem (28). Their solutions included the solitary wave solutions and the periodic wave solutions when the appropriate values of the parameters were particularly selected. Comparing the mathematical structures of their specific solutions with our obtained solutions, almost all of their specific solutions can be obtained from our solutions. For instance, solutions (20), (23), and (24) in their paper are structurally equivalent to the hyperbolic form of the solution in (39) via using $C=D=1$ and $C=1, D=-1$. The trigonometric solution (28) in their paper has the same mathematical structure as our solution in (38) with $C=D=1$ and $C=-1, D=1$. Solutions (29), (32), and (33) in their article can be equivalently transformed to our solution in (38) by choosing $C=0, D=1$ and $C=1, D=0$. Finally, rational solutions (36) and (37) in their paper with $\xi$ of degree two in the denominators are structurally equivalent to our rational solution in (40). Furthermore, the exact solutions in (41) and (42) can generate other types of solutions different from the compared ones.

In $[46,47]$, they used the estimated dimensionless parameter $\sigma=1.67 \times 10^{2}$ and took $\sigma c=2.5,2 \delta-w\left(G_{0} / C_{0}\right)=0.1$ for plotting their solutions. Using the parameter values mentioned above and choosing $\mu=0.5, \lambda=-1, C=1, D=-1$, we obtain the graphical representations of the exact traveling wave solution $u_{2}^{2}(x, t)$ in (39) as demonstrated in Figure 2 describing the soliton solution of bell-type. The obtained three-dimensional graph shown in Figure 2(a) is similar to their results.

3.3. The Generalized Hirota-Satsuma Coupled KdV System. In 1982 Satsuma and Hirota proposed the new system of equations which is called the generalized Hirota-Satsuma coupled KdV system as follows [49]:

$$
\begin{aligned}
U_{t} & =\frac{1}{4} U_{x x x}+3 U U_{x}+3\left(-V^{2}+W\right)_{x} \\
V_{t} & =-\frac{1}{2} V_{x x x}-3 U V_{x} \\
W_{t} & =-\frac{1}{2} W_{x x x}-3 U W_{x} .
\end{aligned}
$$

The above system can be obtained from the four reductions of KP hierarchy. In particular, the well-known Hirota-Satsuma coupled KdV system [50], which was derived in 1981 by Hirota and Satsuma to describe interactions of two long waves with different dispersion relations, can be obtained by setting $W=0$ in (44). We want to obtain traveling wave solutions for system (44) which are in the following form:

$$
\begin{aligned}
U(x, t) & =u(\xi), \\
V(x, t) & =v(\xi), \\
W(x, t) & =w(\xi), \\
\xi & =k(x-c t),
\end{aligned}
$$

where $k$ and $c$ are nonzero arbitrary constants to be determined later. Substituting (45) into (44), we yield a system of ODEs as follows:

$$
-c k u^{\prime}=\frac{1}{4} k^{3} u^{\prime \prime \prime}+3 k u u^{\prime}+3 k\left(-v^{2}+w\right)^{\prime},
$$




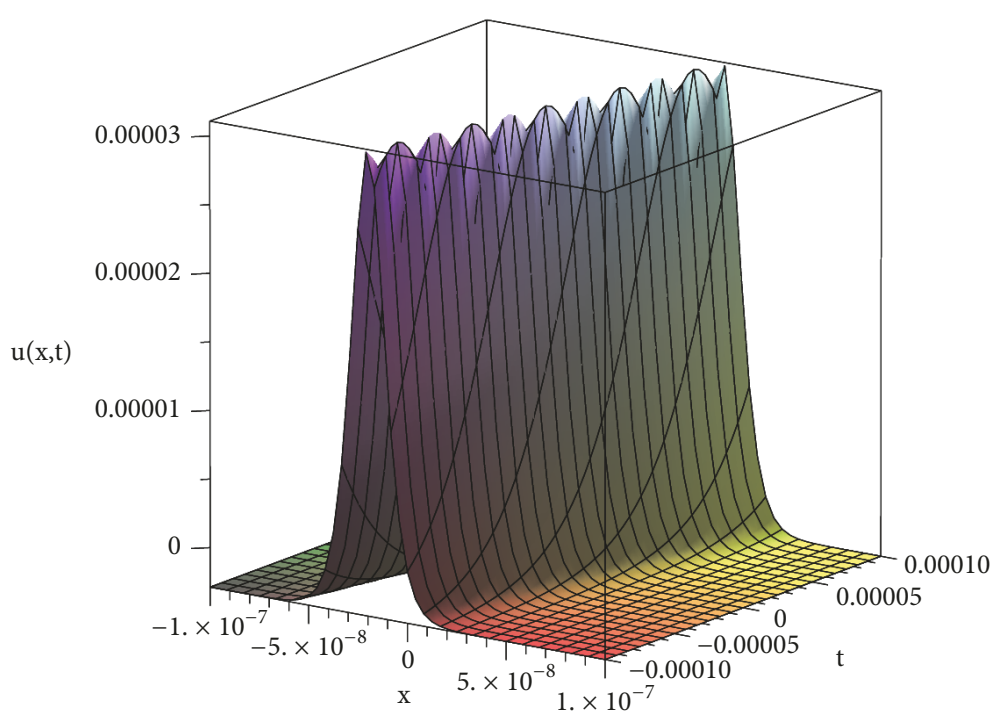

(a) $3 \mathrm{D}$ graph

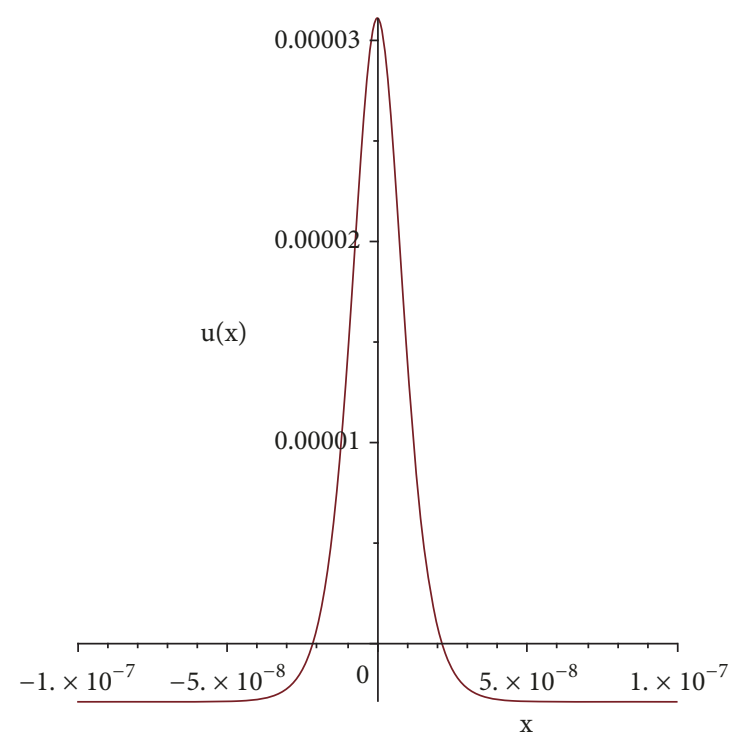

(b) $2 \mathrm{D}$ graph when $t=0$

FIGURE 2: The soliton solution of bell-type for $u_{2}^{2}(x, t)$ in (39) when $\alpha=1.8 \times 10^{7}, \beta=500.4, c=0.01497, \mu=0.5, \lambda=-1, C=1$, and $D=-1$.

$$
\begin{aligned}
& -c k v^{\prime}=-\frac{1}{2} k^{3} v^{\prime \prime \prime}-3 k u v^{\prime}, \\
& -c k w^{\prime}=-\frac{1}{2} k^{3} w^{\prime \prime \prime}-3 k u w^{\prime} .
\end{aligned}
$$

Let [51]

$$
\begin{aligned}
& u=\alpha v^{2}+\beta v+\gamma \\
& w=A v+B
\end{aligned}
$$

where $\alpha, \beta, \gamma, A$, and $B$ are constants to be also determined later.

Substituting (49) into (47) and (48) and then integrating once, we know that (47) and (48) give the same equation as follows:

$$
k^{2} v^{\prime \prime}=-2 \alpha v^{3}-3 \beta v^{2}+2(c-3 \gamma) v+c_{1},
$$

where $c_{1}$ is a constant of integration. Multiplying (50) by $v^{\prime}$ and then integrating the resulting equation with respect to $\xi$, we obtain

$$
k^{2}\left(v^{\prime}\right)^{2}=-\alpha v^{4}-2 \beta v^{3}+2(c-3 \gamma) v^{2}+2 c_{1} v+c_{2},
$$

where $c_{2}$ is also a constant of integration.

From (49)-(51), we obtain

$$
\begin{aligned}
k^{2} u^{\prime \prime} & =2 \alpha k^{2} v^{\prime 2}+k^{2}(2 \alpha v+\beta) v^{\prime \prime}, \\
= & 2 \alpha\left[-\alpha v^{4}-2 \beta v^{3}+2(c-3 \gamma) v^{2}+2 c_{1} v+c_{2}\right] \\
& +(2 \alpha v+\beta)\left[-2 \alpha v^{3}-3 \beta v^{2}+2(c-3 \gamma) v+c_{1}\right] .
\end{aligned}
$$

Integrating (46) once, we get

$$
\frac{1}{4} k^{2} u^{\prime \prime}+\frac{3}{2} u^{2}+c u+3\left(-v^{2}+w\right)+c_{3}=0,
$$

where $c_{3}$ is a constant of integration. Substituting (49) and (52) into (53), we yield the following system:

$$
\begin{aligned}
3 \alpha c-3 \alpha \gamma+\frac{3}{4} \beta^{2}-3 & =0, \\
\frac{1}{2}\left(\alpha c_{1}+\beta c+\gamma \beta\right)+A & =0, \\
\frac{1}{4}\left(2 \alpha c_{2}+\beta c_{1}\right)+\frac{3}{2} \gamma^{2}+c \gamma+3 B+c_{3} & =0 .
\end{aligned}
$$

Let

$$
\begin{aligned}
c_{1} & =\frac{1}{2 \alpha^{2}}\left(\beta^{3}+2 c \alpha \beta-6 \alpha \beta \gamma\right), \\
v(\xi) & =a P(\xi)-\frac{\beta}{2 \alpha} .
\end{aligned}
$$

We find from (54) that

$$
\begin{aligned}
\alpha= & \frac{\beta^{2}-4}{4(\gamma-c)}, \\
A= & \frac{4 \beta(c-\gamma)}{\beta^{2}-4}, \\
B= & \frac{1}{6(-\gamma+c)\left(\beta^{2}-4\right)^{2}}\left(16 c_{3} c \beta^{2}-2 c_{3} c \beta^{4}\right. \\
& -16 c_{3} \gamma \beta^{2}+2 c_{3} \gamma \beta^{4}+56 c^{2} \gamma \beta^{2}-48 \gamma^{2} c \beta^{2}-16 c_{2} \\
& +\frac{1}{4} c_{2} \beta^{6}-3 c_{2} \beta^{4}+12 c_{2} \beta^{2}-16 \gamma^{2} c-32 c^{2} \gamma \\
& -8 c^{3} \beta^{2}+\beta^{4} \gamma^{3}-2 \beta^{4} c^{3}+32 c_{3} \gamma-32 c_{3} c+48 \gamma^{3} \\
& \left.+\beta^{4} \gamma^{2} c\right) .
\end{aligned}
$$


From (50), we therefore obtain

$$
a k^{2} P^{\prime \prime}-a\left(2 c-6 \gamma+\frac{3 \beta^{2}}{2 \alpha}\right) P+2 \alpha a^{3} P^{3}=0
$$

Applying the homogeneous balance principle and (6) mentioned in Step 3 to the terms $P^{\prime \prime}$ and $P^{3}$, we then have that

$$
\operatorname{deg}\left[P^{\prime \prime}\right]=N+2=\operatorname{deg}\left[P^{3}\right]=3 N,
$$

which leads to $N=1$. Hence, the form of exact solutions of the ordinary differential equation in (57) using the $\left(G^{\prime} / G^{2}\right)$ expansion method is

$$
P(\xi)=a_{0}+a_{1}\left(\frac{G^{\prime}}{G^{2}}\right)+b_{1}\left(\frac{G^{\prime}}{G^{2}}\right)^{-1},
$$

where $a_{0}, a_{1}, b_{1}$ are unknown constants. Substituting (59) into (57) along with (5), then collecting all the coefficients with the same power of $\left(G^{\prime} / G^{2}\right)^{i},(i=0, \pm 1, \pm 2, \ldots)$, and finally setting these resulting coefficients to be zero, we consequently attain the following system of algebraic equations in $a_{0}, a_{1}, b_{1}, k, c, a, \alpha, \beta, \gamma$ :

$$
\begin{aligned}
& \left(\frac{G^{\prime}}{G^{2}}\right)^{-3}: 2 \alpha a^{3} b_{1}^{3}+2 a b_{1} k^{2} \mu^{2}=0, \\
& \left(\frac{G^{\prime}}{G^{2}}\right)^{-2}: 6 \alpha a_{0} a^{3} b_{1}^{2}=0, \\
& \left(\frac{G^{\prime}}{G^{2}}\right)^{-1}: 2 a b_{1} \mu \lambda k^{2}-\frac{3 a b_{1} \beta^{2}}{2 \alpha}-2 a c b_{1}+6 a \gamma b_{1} \\
& +6 \alpha b_{1} a^{3} a_{0}^{2}+6 \alpha a_{1} a^{3} b_{1}^{2}=0, \\
& \left(\frac{G^{\prime}}{G^{2}}\right)^{0}:-\frac{3 a \beta^{2} a_{0}}{2 \alpha}-2 a c a_{0}+6 a \gamma a_{0}+2 \alpha a^{3} a_{0}^{3} \\
& +12 \alpha a^{3} a_{0} a_{1} b_{1}=0, \\
& \left(\frac{G^{\prime}}{G^{2}}\right)^{1}: 2 k^{2} a a_{1} \lambda \mu-\frac{3 a \beta^{2} a_{1}}{2 \alpha}-2 a c a_{1}+6 a \gamma a_{1} \\
& +6 \alpha a^{3} a_{0}^{2} a_{1}+6 \alpha a^{3} a_{1}^{2} b_{1}=0, \\
& \left(\frac{G^{\prime}}{G^{2}}\right)^{2}: 6 \alpha a^{3} a_{0} a_{1}^{2}=0 \text {, } \\
& \left(\frac{G^{\prime}}{G^{2}}\right)^{3}: 2 a^{3} \alpha a_{1}^{3}+2 a k^{2} \lambda^{2} a_{1}=0 .
\end{aligned}
$$

By solving the nonlinear system in (60) with Maple, we obtain the following cases.

Case 1.

$$
\begin{aligned}
& a_{0}=0, \\
& a_{1}=0, \\
& b_{1}= \pm \frac{\sqrt{-1 / \alpha} \mu k}{a}
\end{aligned}
$$

$$
\begin{aligned}
& k=k, \\
& c=\frac{1}{4 \alpha}\left(4 k^{2} \lambda \mu \alpha+12 \alpha \gamma-3 \beta^{2}\right),
\end{aligned}
$$

where $k, a \neq 0, \beta, \gamma, \mu, \lambda$ are arbitrary constants, and $\alpha \neq 0$ is expressed in (56).

Case 2.

$$
\begin{aligned}
a_{0} & =0, \\
a_{1} & = \pm \frac{\sqrt{-1 / \alpha} \lambda k}{a}, \\
b_{1} & =0, \\
k & =k, \\
c & =\frac{1}{4 \alpha}\left(4 k^{2} \lambda \mu \alpha+12 \alpha \gamma-3 \beta^{2}\right),
\end{aligned}
$$

where $k, a \neq 0, \beta, \gamma, \mu, \lambda$ are arbitrary constants, and $\alpha \neq 0$ is expressed in (56).

Case 3.

$$
\begin{aligned}
& a_{0}=0, \\
& a_{1}= \pm \frac{\sqrt{-1 / \alpha} \lambda k}{a}, \\
& b_{1}= \pm \frac{\sqrt{-1 / \alpha} \mu k}{a}, \\
& k=k \\
& c=\frac{1}{4 \alpha}\left(-8 k^{2} \lambda \mu \alpha+12 \alpha \gamma-3 \beta^{2}\right),
\end{aligned}
$$

where $k, a \neq 0, \beta, \gamma, \mu, \lambda$ are arbitrary constants, and $\alpha \neq 0$ is expressed in (56).

Case 4.

$$
\begin{aligned}
& a_{0}=0, \\
& a_{1}= \pm \frac{\sqrt{-1 / \alpha} \lambda k}{a} \\
& b_{1}=\mp \frac{\sqrt{-1 / \alpha} \mu k}{a} \\
& k=k \\
& c=\frac{1}{4 \alpha}\left(16 k^{2} \lambda \mu \alpha+12 \alpha \gamma-3 \beta^{2}\right),
\end{aligned}
$$

where $k, a \neq 0, \beta, \gamma, \mu, \lambda$ are arbitrary constants, and $\alpha \neq 0$ is expressed in (56).

Inserting the above four cases shown in (61)-(64) along with the functions $G^{\prime} / G^{2}$ described in (7)-(10) into the solution form (59), we obtain four results of solutions of system (44) as follows. 
Result 1. Using parameter values specified in Case 1 as shown in $(61)$ and $\xi=k\left(x-(1 / 4 \alpha)\left(4 k^{2} \lambda \mu \alpha+12 \alpha \gamma-3 \beta^{2}\right) t\right)$, we have the following exact solutions.

When $\mu \lambda>0$, the trigonometric function solution of system (44) can be written as

$$
\begin{aligned}
& v_{1}^{1}(x, t) \\
& = \pm k \sqrt{-\frac{\mu \lambda}{\alpha}}\left(\frac{C \cos (\sqrt{\mu \lambda} \xi)+D \sin (\sqrt{\mu \lambda} \xi)}{D \cos (\sqrt{\mu \lambda} \xi)-C \sin (\sqrt{\mu \lambda} \xi)}\right)^{-1} \\
& -\frac{\beta}{2 \alpha} \\
& u_{1}^{1}(x, t)=\alpha\left(v_{1}^{1}(x, t)\right)+\beta\left(v_{1}^{1}(x, t)\right)+\gamma \\
& w_{1}^{1}(x, t)=A\left(v_{1}^{1}(x, t)\right)+B .
\end{aligned}
$$

When $\mu \lambda<0$, the exponential function solution of system (44) can be expressed as

$$
\begin{aligned}
& v_{2}^{1}(x, t) \\
& = \pm 2 \mu \lambda k \sqrt{-\frac{1}{\alpha}}\left(2 \sqrt{|\mu \lambda|}-\frac{4 C \sqrt{|\mu \lambda| e^{2 \xi} \sqrt{|\mu \lambda|}}}{C e^{2 \xi \sqrt{|\mu \lambda|}}-D}\right)^{-1} \\
& -\frac{\beta}{2 \alpha} \\
& u_{2}^{1}(x, t)=\alpha\left(v_{2}^{1}(x, t)\right)+\beta\left(v_{2}^{1}(x, t)\right)+\gamma \\
& w_{2}^{1}(x, t)=A\left(v_{2}^{1}(x, t)\right)+B .
\end{aligned}
$$

When $\mu=0, \lambda \neq 0$, the rational function solution of system (44) can be written as

$$
\begin{aligned}
& v_{3}^{1}(x, t)=-\frac{\beta}{2 \alpha}, \\
& u_{3}^{1}(x, t)=\alpha\left(v_{3}^{1}(x, t)\right)+\beta\left(v_{3}^{1}(x, t)\right)+\gamma, \\
& w_{3}^{1}(x, t)=A\left(v_{3}^{1}(x, t)\right)+B .
\end{aligned}
$$

Result 2. Using parameter values specified in Case 2 as shown in (62) and $\xi=k\left(x-(1 / 4 \alpha)\left(4 k^{2} \lambda \mu \alpha+12 \alpha \gamma-3 \beta^{2}\right) t\right)$, we have the following exact solutions.

When $\mu \lambda>0$, the trigonometric function solution of system (44) can be written as

$$
\begin{aligned}
& v_{1}^{2}(x, t) \\
& = \pm k \sqrt{-\frac{\mu \lambda}{\alpha}}\left(\frac{C \cos (\sqrt{\mu \lambda} \xi)+D \sin (\sqrt{\mu \lambda} \xi)}{D \cos (\sqrt{\mu \lambda} \xi)-C \sin (\sqrt{\mu \lambda} \xi)}\right) \\
& -\frac{\beta}{2 \alpha}, \\
& u_{1}^{2}(x, t)=\alpha\left(v_{1}^{2}(x, t)\right)+\beta\left(v_{1}^{2}(x, t)\right)+\gamma, \\
& w_{1}^{2}(x, t)=A\left(v_{1}^{2}(x, t)\right)+B .
\end{aligned}
$$

When $\mu \lambda<0$, the exponential function solution of system (44) can be expressed as

$$
\begin{aligned}
v_{2}^{2}(x, t)= & \pm \frac{k}{2} \sqrt{-\frac{1}{\alpha}}\left(2 \sqrt{|\mu \lambda|}-\frac{4 C \sqrt{|\mu \lambda|} e^{2 \xi \sqrt{|\mu \lambda|}}}{C e^{2 \xi \sqrt{|\mu \lambda|}}-D}\right) \\
& -\frac{\beta}{2 \alpha} \\
u_{2}^{2}(x, t)= & \alpha\left(v_{2}^{2}(x, t)\right)+\beta\left(v_{2}^{2}(x, t)\right)+\gamma, \\
w_{2}^{2}(x, t)= & A\left(v_{2}^{2}(x, t)\right)+B .
\end{aligned}
$$

When $\mu=0, \lambda \neq 0$, the rational function solution of system (44) can be written as

$$
\begin{aligned}
& v_{3}^{2}(x, t)=\mp \frac{k C \sqrt{-1 / \alpha}}{C \xi+D}-\frac{\beta}{2 \alpha}, \\
& u_{3}^{2}(x, t)=\alpha\left(v_{3}^{2}(x, t)\right)+\beta\left(v_{3}^{2}(x, t)\right)+\gamma, \\
& w_{3}^{2}(x, t)=A\left(v_{3}^{2}(x, t)\right)+B,
\end{aligned}
$$

for which the traveling wave transformation for this case is $\xi=k\left(x-\left(6 \gamma /\left(\beta^{2}+2\right)\right) t\right)$.

Result 3. Using parameter values specified in Case 3 as shown in $(63)$ and $\xi=k\left(x-(1 / 4 \alpha)\left(-8 k^{2} \lambda \mu \alpha+12 \alpha \gamma-3 \beta^{2}\right) t\right)$, we have the following exact solutions.

When $\mu \lambda>0$, the trigonometric function solution of system (44) can be written as

$$
\begin{aligned}
& v_{1}^{3}(x, t) \\
& = \pm k \sqrt{-\frac{\mu \lambda}{\alpha}}\left(\frac{C \cos (\sqrt{\mu \lambda} \xi)+D \sin (\sqrt{\mu \lambda} \xi)}{D \cos (\sqrt{\mu \lambda} \xi)-C \sin (\sqrt{\mu \lambda} \xi)}\right) \\
& \pm k \sqrt{-\frac{\mu \lambda}{\alpha}}\left(\frac{C \cos (\sqrt{\mu \lambda} \xi)+D \sin (\sqrt{\mu \lambda} \xi)}{D \cos (\sqrt{\mu \lambda} \xi)-C \sin (\sqrt{\mu \lambda} \xi)}\right) \\
& -\frac{\beta}{2 \alpha}, \\
& u_{1}^{3}(x, t)=\alpha\left(v_{1}^{3}(x, t)\right)+\beta\left(v_{1}^{3}(x, t)\right)+\gamma, \\
& w_{1}^{3}(x, t)=A\left(v_{1}^{3}(x, t)\right)+B .
\end{aligned}
$$

When $\mu \lambda<0$, the exponential function solution of system (44) can be expressed as

$$
\begin{aligned}
& v_{2}^{3}(x, t) \\
& = \pm \frac{k}{2} \sqrt{-\frac{1}{\alpha}}\left(2 \sqrt{|\mu \lambda|}-\frac{4 C \sqrt{|\mu \lambda|} e^{2 \xi \sqrt{|\mu \lambda|}}}{C e^{2 \xi \sqrt{|\mu \lambda|}}-D}\right) \\
& \quad \pm 2 \mu \lambda k \sqrt{-\frac{1}{\alpha}}\left(2 \sqrt{|\mu \lambda|}-\frac{4 C \sqrt{|\mu \lambda|} e^{2 \xi \sqrt{|\mu \lambda|}}}{C e^{2 \xi \sqrt{|\mu \lambda|}}-D}\right)^{-1}
\end{aligned}
$$




$$
\begin{gathered}
-\frac{\beta}{2 \alpha}, \\
u_{2}^{3}(x, t)=\alpha\left(v_{2}^{3}(x, t)\right)+\beta\left(v_{2}^{3}(x, t)\right)+\gamma, \\
w_{2}^{3}(x, t)=A\left(v_{2}^{3}(x, t)\right)+B .
\end{gathered}
$$

When $\mu=0, \lambda \neq 0$, the rational function solution of system (44) can be written as

$$
\begin{aligned}
& v_{3}^{3}(x, t)=\mp \frac{k C \sqrt{-1 / \alpha}}{C \xi+D}-\frac{\beta}{2 \alpha}, \\
& u_{3}^{3}(x, t)=\alpha\left(v_{3}^{3}(x, t)\right)+\beta\left(v_{3}^{3}(x, t)\right)+\gamma, \\
& w_{3}^{3}(x, t)=A\left(v_{3}^{3}(x, t)\right)+B,
\end{aligned}
$$

for which the traveling wave transformation for this case is $\xi=k\left(x-\left(6 \gamma /\left(\beta^{2}+2\right)\right) t\right)$.

Result 4. Using parameter values specified in Case 4 as shown in (64) and $\xi=k\left(x-(1 / 4 \alpha)\left(16 k^{2} \lambda \mu \alpha+12 \alpha \gamma-3 \beta^{2}\right) t\right)$, we have the following exact solutions.

When $\mu \lambda>0$, the trigonometric function solution of system (44) can be written as

$$
\begin{aligned}
& v_{1}^{4}(x, t) \\
& = \pm k \sqrt{-\frac{\mu \lambda}{\alpha}}\left(\frac{C \cos (\sqrt{\mu \lambda} \xi)+D \sin (\sqrt{\mu \lambda} \xi)}{D \cos (\sqrt{\mu \lambda} \xi)-C \sin (\sqrt{\mu \lambda} \xi)}\right) \\
& \mp k \sqrt{-\frac{\mu \lambda}{\alpha}}\left(\frac{C \cos (\sqrt{\mu \lambda} \xi)+D \sin (\sqrt{\mu \lambda} \xi)}{D \cos (\sqrt{\mu \lambda} \xi)-C \sin (\sqrt{\mu \lambda} \xi)}\right)^{-1} \\
& -\frac{\beta}{2 \alpha}, \\
& u_{1}^{4}(x, t)=\alpha\left(v_{1}^{4}(x, t)\right)+\beta\left(v_{1}^{4}(x, t)\right)+\gamma, \\
& w_{1}^{4}(x, t)=A\left(v_{1}^{4}(x, t)\right)+B .
\end{aligned}
$$

When $\mu \lambda<0$, the exponential function solution of system (44) can be expressed as

$$
\begin{aligned}
& v_{2}^{4}(x, t) \\
& = \pm \frac{k}{2} \sqrt{-\frac{1}{\alpha}}\left(2 \sqrt{|\mu \lambda|}-\frac{4 C \sqrt{|\mu \lambda|} e^{2 \xi \sqrt{|\mu \lambda|}}}{C e^{2 \xi \sqrt{|\mu \lambda|}}-D}\right) \\
& \quad \mp 2 \mu \lambda k \sqrt{-\frac{1}{\alpha}}\left(2 \sqrt{|\mu \lambda|}-\frac{4 C \sqrt{|\mu \lambda|} e^{2 \xi \sqrt{|\mu \lambda|}}}{C e^{2 \xi \sqrt{|\mu \lambda|}}-D}\right)^{-1} \\
& \quad-\frac{\beta}{2 \alpha},
\end{aligned}
$$

$$
\begin{aligned}
& u_{2}^{4}(x, t)=\alpha\left(v_{2}^{4}(x, t)\right)+\beta\left(v_{2}^{4}(x, t)\right)+\gamma, \\
& w_{2}^{4}(x, t)=A\left(v_{2}^{4}(x, t)\right)+B .
\end{aligned}
$$

When $\mu=0, \lambda \neq 0$, the rational function solution of system (44) can be written as

$$
\begin{aligned}
& v_{3}^{4}(x, t)=\mp \frac{k C \sqrt{-1 / \alpha}}{C \xi+D}-\frac{\beta}{2 \alpha}, \\
& u_{3}^{4}(x, t)=\alpha\left(v_{3}^{4}(x, t)\right)+\beta\left(v_{3}^{4}(x, t)\right)+\gamma, \\
& w_{3}^{4}(x, t)=A\left(v_{3}^{4}(x, t)\right)+B,
\end{aligned}
$$

for which the traveling wave transformation for this case is $\xi=k\left(x-\left(6 \gamma /\left(\beta^{2}+2\right)\right) t\right)$.

Lu et al. [52] applied the new improved Riccati equation method to system (44) and then found that its exact solutions were expressed in terms of fractions of trigonometric functions and fractions of hyperbolic functions. El-Wakil and Abdou [53] found some exact solutions for system (44) by the modified extended tanh-function method. All of the obtained solutions had the forms of the following functions: tanh, coth, tan, and cot. It is not difficult to check that our exact solutions of system (44), which are shown in (65)-(76), are the functions whose mathematical forms are equivalent to those of most of the solutions found previously by choosing suitable parameters and some of them are obviously novel.

In Figures 3 and 4, we present the three-dimensional and two-dimensional plots of some exact solutions, which are $v_{1}^{4}(x, t)$ and $v_{2}^{4}(x, t)$, expressed in (74) and (75), respectively. They are depicted using the sets of the appropriate parameter values as described below. Employing $c=-7.25, \alpha=$ -0.23077 , which are solved implicitly using (56), (61) and the parameter values $k=0.5, \beta=1, \gamma=-4, \mu=1.5, \lambda=1$, and choosing $C=D=1$, we obtain the plots of the selected exact traveling wave solution

$$
\begin{aligned}
v_{1}^{4}(x, t) & \\
= & -k \sqrt{-\frac{\mu \lambda}{\alpha}}\left[\left(\frac{C \cos (\sqrt{\mu \lambda} \xi)+D \sin (\sqrt{\mu \lambda} \xi)}{D \cos (\sqrt{\mu \lambda} \xi)-C \sin (\sqrt{\mu \lambda} \xi)}\right)\right. \\
& \left.-\left(\frac{C \cos (\sqrt{\mu \lambda} \xi)+D \sin (\sqrt{\mu \lambda} \xi)}{D \cos (\sqrt{\mu \lambda} \xi)-C \sin (\sqrt{\mu \lambda} \xi)}\right)^{-1}\right]-\frac{\beta}{2 \alpha},
\end{aligned}
$$

as shown in Figure 3 which represents the periodic wave solutions.

Again implicitly solving (56) and (61) via using $k=1, \beta=$ 2, $\gamma=2, \mu=2, \lambda=-7 / 8$, we obtain $c=2, \alpha=-1$. Then the graphical representations of the chosen exact solution

$$
\begin{aligned}
v_{2}^{4}(x, t) & =-\frac{k}{2} \sqrt{-\frac{1}{\alpha}}\left(2 \sqrt{|\mu \lambda|}-\frac{4 C \sqrt{|\mu \lambda|} e^{2 \xi \sqrt{|\mu \lambda|}}}{C e^{2 \xi \sqrt{|\mu \lambda|}}-D}\right)
\end{aligned}
$$




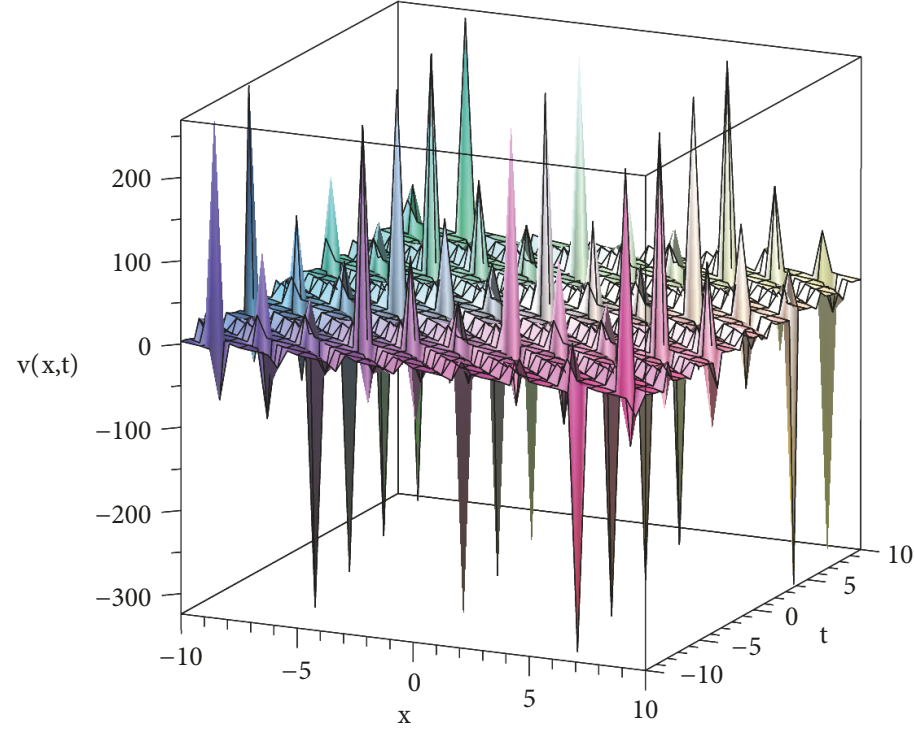

(a) 3D graph

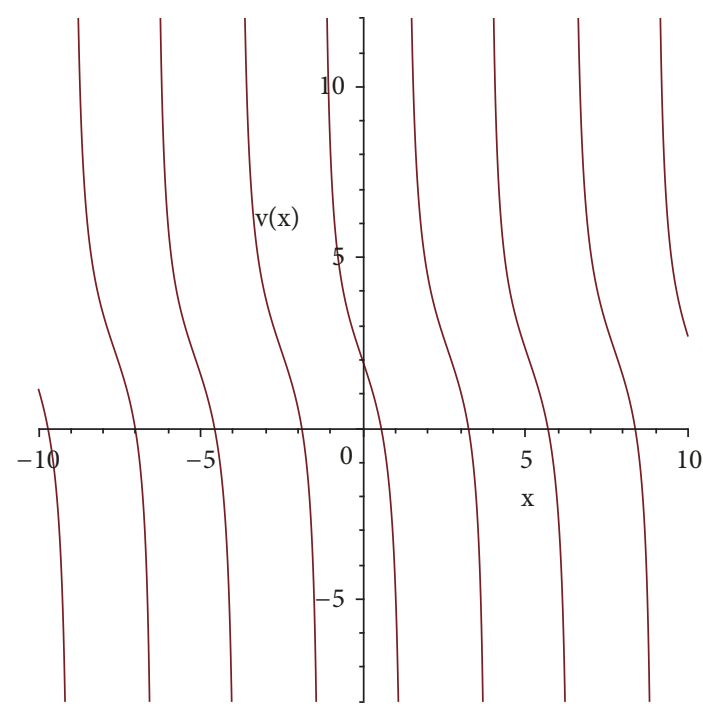

(b) $2 \mathrm{D}$ graph when $t=0$

FIGURE 3: The periodic wave solutions of $v_{1}^{4}(x, t)$ in (77) when $k=0.5, \beta=1, \gamma=-4, \mu=1.5, \lambda=1, c=-7.25, \alpha=-0.23077$, and $C=D=1$.

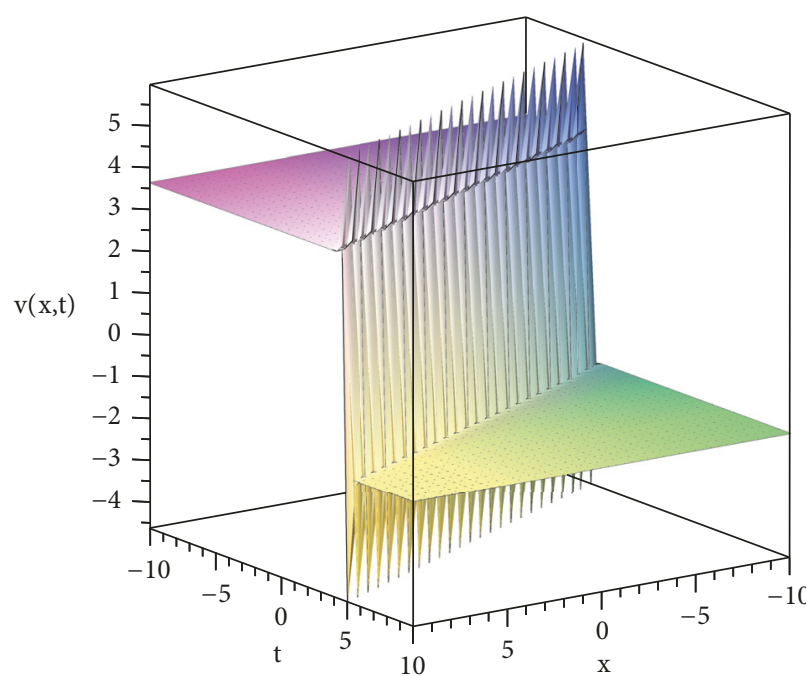

(a) 3D graph

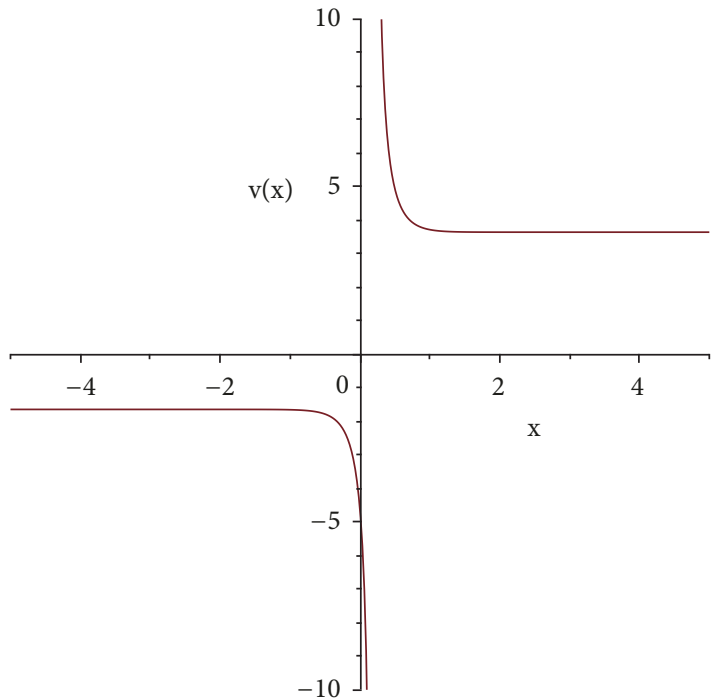

(b) $2 \mathrm{D}$ graph when $t=0$

Figure 4: The singular wave solutions of $v_{2}^{4}(x, t)$ in (78) when $k=1, \beta=2, \gamma=2, \mu=2, \lambda=-7 / 8, c=2, \alpha=-1, C=-3$, and $D=5$.

$$
\begin{aligned}
& +2 \mu \lambda k \sqrt{-\frac{1}{\alpha}}\left(2 \sqrt{|\mu \lambda|}-\frac{4 C \sqrt{|\mu \lambda|} e^{2 \xi \sqrt{|\mu \lambda|}}}{C e^{2 \xi \sqrt{|\mu \lambda|}}-D}\right)^{-1} \\
& -\frac{\beta}{2 \alpha}
\end{aligned}
$$

using the parameters as mentioned above and $C=-3, D=5$ are simulated in Figure 4 describing the singular wave solutions.

\section{Conclusions}

In this paper, the $\left(G^{\prime} / G^{2}\right)$-expansion method has been applied to find some new forms of the explicit exact solutions of the three problems, i.e., the Benny-Luke equation, the equation of nanoionic currents along microtubules, and the generalized Hirota-Satsuma coupled KdV system. The explicit exact solutions of the problems, which are obtained by the method, can be considered as a part of the gigantic variety of possible solution forms; however, they have provided physical representations in each problem. As shown in 
Section 3, we have found that the obtained exact solutions of the problems are expressed in terms of trigonometric, exponential (or equivalently hyperbolic), and rational functions. The investigation demonstrates that the method is considerably efficient and practically appropriate for analytically solving such problems with the aid of Maple. All of the obtained exact solutions of each problem have ensured the correctness by substituting them back into the original equations. Moreover, the method could also be employed efficiently for a broad range of NPDEs of integer orders.

\section{Data Availability}

The data used to support the findings of this study are available from the corresponding author upon request.

\section{Conflicts of Interest}

The authors declare that they have no conflicts of interest.

\section{Acknowledgments}

This research was funded by King Mongkut's University of Technology North Bangkok (Contract no. KMUTNB-KNOW61-037).

\section{References}

[1] D. Lu, A. Seadawy, and M. Arshad, "Applications of extended simple equation method on unstable nonlinear Schrödinger equations," Optik - International Journal for Light and Electron Optics, vol. 140, pp. 136-144, 2017.

[2] M. H. Bazyar and C. Song, "Analysis of transient wave scattering and its applications to site response analysis using the scaled boundary finite-element method," Soil Dynamics and Earthquake Engineering, vol. 98, pp. 191-205, 2017.

[3] S. Kumar, "A new fractional modeling arising in engineering sciences and its analytical approximate solution," Alexandria Engineering Journal, vol. 52, no. 4, pp. 813-819, 2013.

[4] V. F. Nikitin, N. N. Smirnov, M. N. Smirnova, and V. V. Tyurenkova, "On board electronic devices safety subject to high frequency electromagnetic radiation effects," Acta Astronautica, vol. 135, pp. 181-186, 2017.

[5] Siraj-ul-Islam and I. Ahmad, "Local meshless method for PDEs arising from models of wound healing," Applied Mathematical Modelling: Simulation and Computation for Engineering and Environmental Systems, vol. 48, pp. 688-710, 2017.

[6] E. M. Zayed and K. A. Gepreel, "Some applications of the G'Gexpansion method to non-linear partial differential equations," Applied Mathematics and Computation, vol. 212, no. 1, pp. 1-13, 2009.

[7] E. M. Zayed and S. Al-Joudi, "Applications of an extended G'Gexpansion method to find exact solutions of nonlinear PDEs in mathematical physics," Mathematical Problems in Engineering, Article ID 768573, Art. ID 768573, 19 pages, 2010.

[8] E. M. E. Zayed and M. A. M. Abdelaziz, "Travelling wave solutions for the burgers equation and the korteweg-de vries equation with variable coefficients using the generalized (G'G)expansion method," Zeitschrift fur Naturforschung - Section A Journal of Physical Sciences, vol. 65, no. 12, pp. 1065-1070, 2010.
[9] E. M. E. Zayed and M. A. M. Abdelaziz, "The two-variable ( $G^{\prime}$ / $G, 1 / G)$-expansion method for solving the nonlinear KDVmKDV Equation," Mathematical Problems in Engineering, vol. 2012, Article ID 725061, 14 pages, 2012.

[10] E. M. Zayed, A. M. Shahoot, and K. A. Alurrfi, “The G'G, 1Gexpansion method and its applications for constructing many new exact solutions of the higher-order nonlinear Schrödinger equation and the quantum ZakharovKuznetsov equation," Optical and Quantum Electronics, vol. 50, no. 2, p. 96, 2018.

[11] E. M. Zayed and K. A. Alurrfi, “The G'G,1G-expansion method and its applications to two nonlinear Schrödinger equations describing the propagation of femtosecond pulses in nonlinear optical fibers," Optik-International Journal for Light and Electron Optics, vol. 127, no. 4, pp. 1581-1589, 2016.

[12] S. Sirisubtawee, S. Koonprasert, C. Khaopant, and W. Porka, "Two reliable methods for solving the $(3+1)$-dimensional spacetime fractional Jimbo-Miwa equation," Mathematical Problems in Engineering, vol. 30, Article ID 9257019, 2017.

[13] D. Baldwin, Ü. Göktas, and W. Hereman, "Symbolic computation of hyperbolic tangent solutions for nonlinear differentialdifference equations," Computer Physics Communications, vol. 162, no. 3, pp. 203-217, 2004.

[14] I. Aslan, "On the application of the Exp-function method to the KP equation for N-soliton solutions," Applied Mathematics and Computation, vol. 219, no. 6, pp. 2825-2828, 2012.

[15] L. Ravi, S. Saha Ray, and S. Sahoo, "New exact solutions of coupled Boussinesq-Burgers equations by exp-function method," Journal of Ocean Engineering and Science, vol. 2, no. 1, pp. 3446, 2017.

[16] Y. Shi, Z. Dai, and D. Li, "The correct traveling wave solutions for the high-order dispersive nonlinear Schrödinger equation," Applied Mathematics and Computation, vol. 216, no. 5, pp. 15831591, 2010.

[17] A.-M. Wazwaz, "The Hirota's direct method and the tanhcoth method for multiple-soliton solutions of the SawadaKotera-Itö seventh-order equation," Applied Mathematics and Computation, vol. 199, no. 1, pp. 133-138, 2008.

[18] A. Wazwaz, "The Hirota's direct method for multiple-soliton solutions for three model equations of shallow water waves," Applied Mathematics and Computation, vol. 201, no. 1-2, pp. 489-503, 2008.

[19] M. B. Hubert, G. Betchewe, S. Y. Doka, and K. Timoleon Crepin, "Soliton wave solutions for the nonlinear transmission line using the Kudryashov method and the G'G-expansion method," Applied Mathematics and Computation, vol. 239, pp. 299-309, 2014.

[20] P. N. Ryabov, D. I. Sinelshchikov, and M. B. Kochanov, "Application of the Kudryashov method for finding exact solutions of the high order nonlinear evolution equations," Applied Mathematics and Computation, vol. 218, no. 7, pp. 3965-3972, 2011.

[21] E. M. E. Zayed and M. E. M. Elshater, "Jacobi elliptic solutions, soliton solutions and other solutions to four higher-order nonlinear Schrodinger equations using two mathematical methods," Optik - International Journal for Light and Electron Optics, vol. 131, pp. 1044-1062, 2017.

[22] R. A. Van Gorder, "The variational iteration method is a special case of the homotopy analysis method," Applied Mathematics Letters, vol. 45, pp. 81-85, 2015.

[23] A.-M. Wazwaz, "The variational iteration method: A reliable analytic tool for solving linear and nonlinear wave equations," Computers \& Mathematics with Applications, vol. 54, no. 7-8, pp. 895-902, 2007. 
[24] I. Hashim, "Adomian decomposition method for solving BVPs for fourth-order integro-differential equations," Journal of Computational and Applied Mathematics, vol. 193, no. 2, pp. 658-664, 2006.

[25] K. C. Basak, P. C. Ray, and R. K. Bera, "Solution of non-linear Klein-Gordon equation with a quadratic non-linear term by Adomian decomposition method," Communications in Nonlinear Science and Numerical Simulation, vol. 14, no. 3, pp. 718-723, 2009.

[26] P. K. Bera and T. Sil, "Homotopy perturbation method in quantum mechanical problems," Applied Mathematics and Computation, vol. 219, no. 6, pp. 3272-3278, 2012.

[27] H. Saberi Nik, S. Effati, and M. Shirazian, "An approximateanalytical solution for the Hamilton-Jacobi-Bellman equation via homotopy perturbation method," Applied Mathematical Modelling: Simulation and Computation for Engineering and Environmental Systems, vol. 36, no. 11, pp. 5614-5623, 2012.

[28] M. S. Mohamed and K. A. Gepreel, "Reduced differential transform method for nonlinear integral member of KadomtsevPetviashvili hierarchy differential equations," Journal of the Egyptian Mathematical Society, vol. 25, no. 1, pp. 1-7, 2017.

[29] L. Gavete, F. Ureña, J. J. Benito, A. García, M. Ureña, and E. Salete, "Solving second order non-linear elliptic partial differential equations using generalized finite difference method," Journal of Computational and Applied Mathematics, vol. 318, pp. 378-387, 2017.

[30] J. Jose, S.-J. Choi, K. E. T. Giljarhus, and O. T. Gudmestad, "A comparison of numerical simulations of breaking wave forces on a monopile structure using two different numerical models based on finite difference and finite volume methods," Ocean Engineering, vol. 137, pp. 78-88, 2017.

[31] M. Agrawal and C. S. Jog, "A quadratic time finite element method for nonlinear elastodynamics within the context of hybrid finite elements," Applied Mathematics and Computation, vol. 305, pp. 203-220, 2017.

[32] J.-K. Zhang, H. Dong, E.-Z. Zhou, B.-W. Li, and X.-Y. Tian, "A combined method for solving 2D incompressible flow and heat transfer by spectral collocation method and artificial compressibility method," International Journal of Heat and Mass Transfer, vol. 112, pp. 289-299, 2017.

[33] Y. Gao and L. Mei, "Galerkin finite element methods for twodimensional RLW and SRLW equations," Applicable Analysis: An International Journal, pp. 1-25, 2017.

[34] J. P. Chen and H. Chen, “The G'G2-expansion method and its application to coupled nonlinear Klein-Gordon equation," Journal of South China Normal University, vol. 44, no. 2, pp. 63-66, 2012.

[35] L. Wen-An, C. Hao, and Z. Guo-Cai, "The ( $\omega / \mathrm{g})$-expansion method and its application to Vakhnenko equation," Chinese Physics B, vol. 18, no. 2, pp. 400-404, 2009.

[36] E. M. Zayed and A. H. Arnous, "The modified wg-expansion method and its applications for solving the modified generalized Vakhnenko equation," Italian Journal of Pure and Applied Mathematics, no. 32, pp. 477-492, 2014.

[37] M. Wang, X. Li, and J. Zhang, “The G'G-expansion method and travelling wave solutions of nonlinear evolution equations in mathematical physics," Physics Letters A, vol. 372, no. 4, pp. 417423, 2008.

[38] Z. Kang, "G'G2-expansion solutions to MBBM and OBBM equations," Journal of Partial Differential Equations, vol. 28, no. 2, pp. 158-166, 2015.
[39] H. K. Rafsanjani, M. H. Sedaaghi, and S. Saryazdi, "Efficient diffusion coefficient for image denoising," Computers \& Mathematics with Applications, vol. 72, no. 4, pp. 893-903, 2016.

[40] S. T. Mohyud-Din and S. Bibi, "Exact solutions for nonlinear fractional differential equations using G'G2-expansion method," Alexandria Engineering Journal, 2017.

[41] Y. Zhang, L. Zhang, and J. Pang, "Application of G'G2 expansion method for solving Schr $\div$ dingers equation with three-order dispersion," Advances in Applied Mathematics, vol. 6, pp. 212217, 2017

[42] M. N Alam, M. A. Akbar, and S. T. Mohyud-Din, "A novel (G'G)expansion method and its application to the Boussinesq equation," Chinese Physics B, vol. 23, no. 2, Article ID 020203, 2013.

[43] M. N. Alam and M. A. Akbar, "Traveling wave solutions of the nonlinear (1+1)-dimensional modified Benjamin-Bona-Mahony equation by using novel (G'G)-expansion method," Physical Review \& Research International, vol. 4, no. 1, pp. 147-165, 2014.

[44] J. Akter and M. Ali Akbar, "Exact solutions to the Benney-Luke equation and the Phi- 4 equations by using modified simple equation method," Results in Physics, vol. 5, pp. 125-130, 2015.

[45] M. S. Islam, K. Khan, and M. A. Akbar, "Application of the improved $F$-expansion method with Riccati equation to find the exact solution of the nonlinear evolution equations," Journal of the Egyptian Mathematical Society, vol. 25, no. 1, pp. 13-18, 2017.

[46] M. V. Satarić, D. Sekulić, and M. Živanov, "Solitonic ionic currents along microtubules," Journal of Computational and Theoretical Nanoscience, vol. 7, no. 11, pp. 2281-2290, 2010.

[47] D. L. Sekulic, M. V. Sataric, and M. B. Zivanov, "Symbolic computation of some new nonlinear partial differential equations of nanobiosciences using modified extended tanh-function method," Applied Mathematics and Computation, vol. 218, no. 7, pp. 3499-3506, 2011.

[48] E. M. Zayed and K. A. Alurrfi, “The G'G,1G-expansion method and its applications to find the exact solutions of nonlinear PDEs for nanobiosciences," Mathematical Problems in Engineering, Article ID 521712, Art. ID 521712, 10 pages, 2014.

[49] J. Satsuma and R. Hirota, "A coupled KdV equation is one case of the four-reduction of the KP hierarchy," Journal of the Physical Society of Japan, vol. 51, no. 10, pp. 3390-3397, 1982.

[50] R. Hirota and J. Satsuma, "Soliton solutions of a coupled Korteweg-de Vries equation," Physics Letters A, vol. 85, no. 8-9, pp. 407-408, 1981.

[51] Z. Yan, "The extended Jacobian elliptic function expansion method and its application in the generalized Hirota-Satsuma coupled KdV system," Chaos, Solitons \& Fractals, vol. 15, no. 3, pp. 575-583, 2003.

[52] D. Lu, B. Hong, and L. Tian, "New explicit exact solutions for the generalized coupled Hirota- Satsuma KdV system," Computers Mathematics with Applications, vol. 53, no. 8, Article ID 10.1016/j.camwa.2006.08.047, pp. 1181-1190, 2007.

[53] S. A. El-Wakil and M. A. Abdou, "New exact travelling wave solutions using modified extended tanh-function method," Chaos, Solitons \& Fractals, vol. 31, no. 4, pp. 840-852, 2007. 


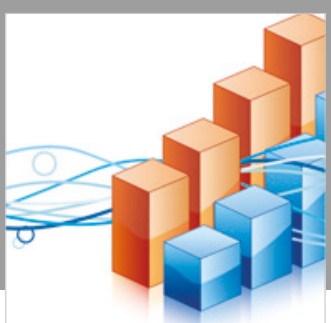

Advances in

Operations Research

\section{-n-m}
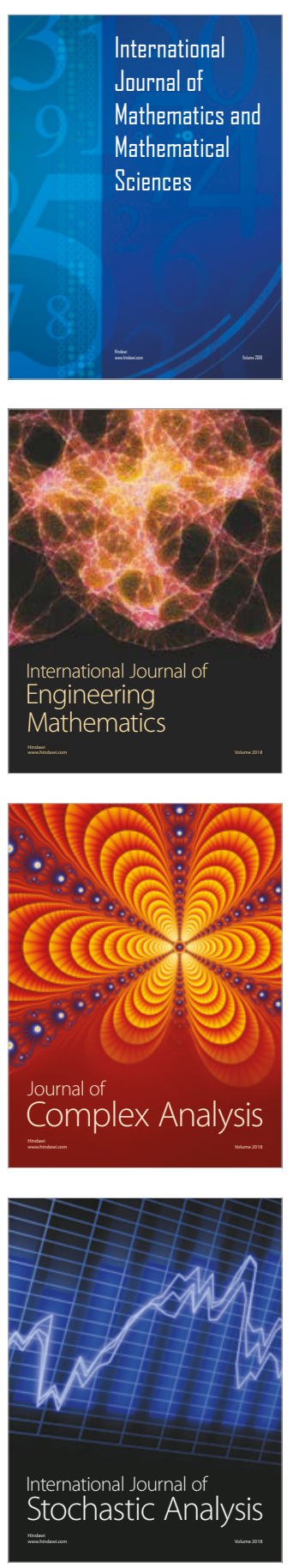
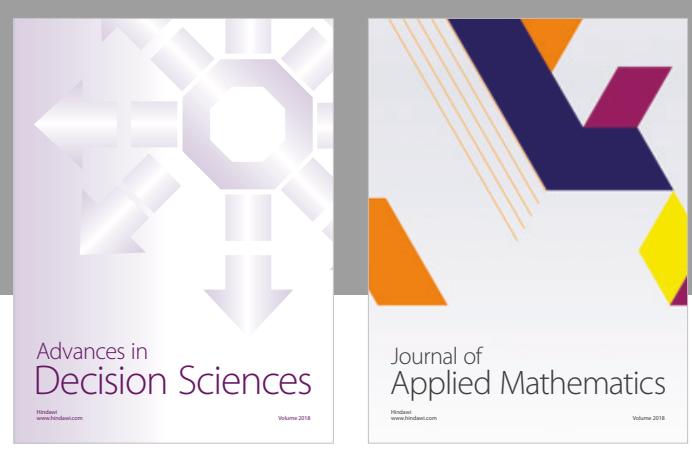

Journal of

Applied Mathematics
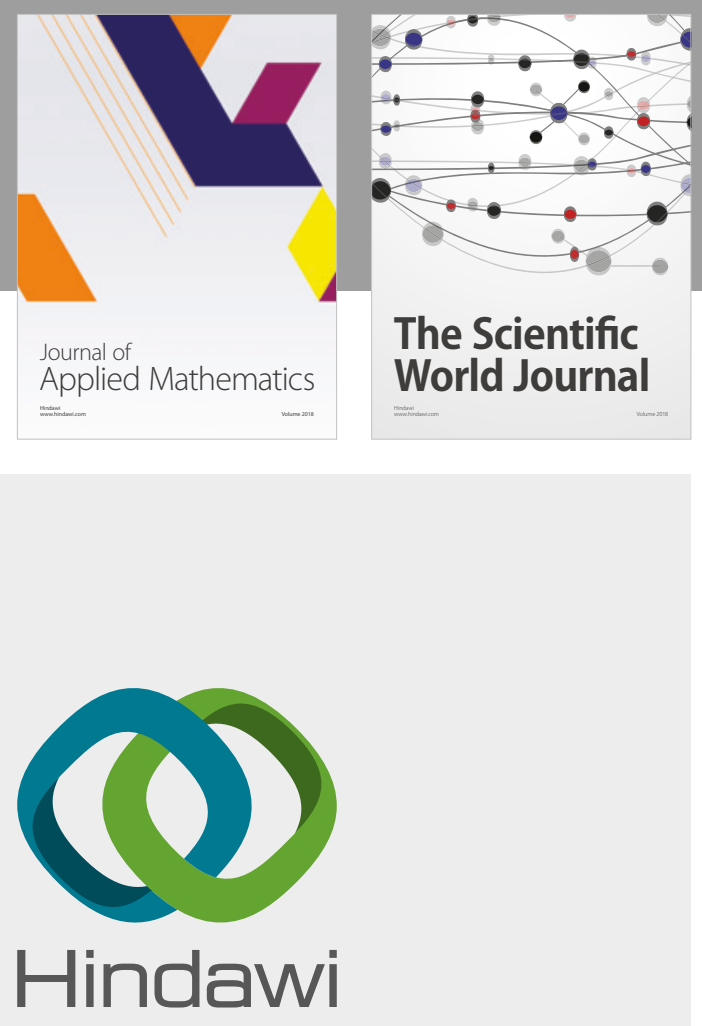

Submit your manuscripts at

www.hindawi.com

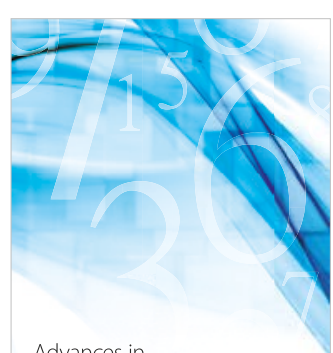

Advances in
Numerical Analysis
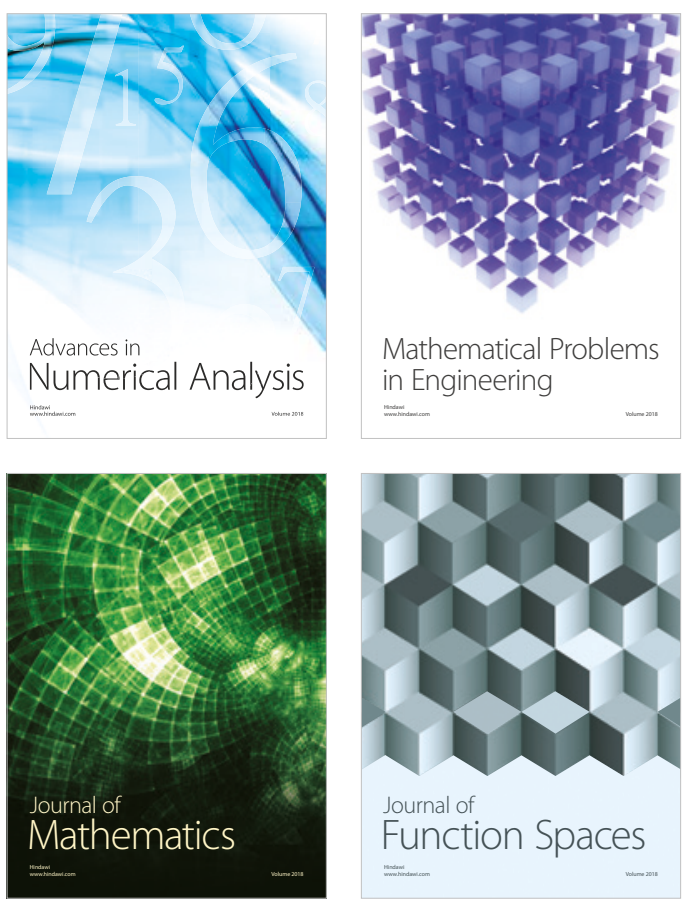

Mathematical Problems in Engineering

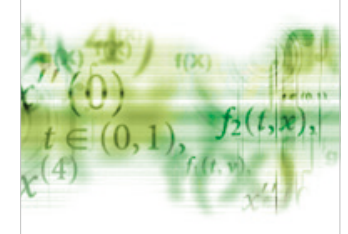

International Journal of

Differential Equations

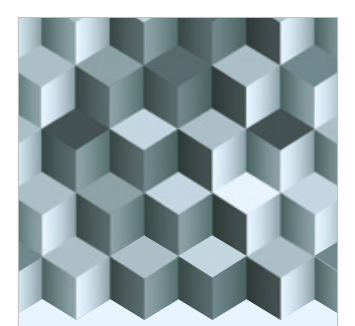

Journal of

Function Spaces

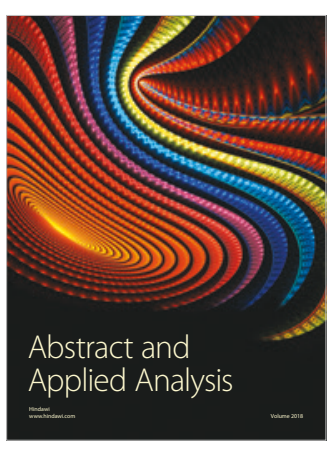

The Scientific

World Journal

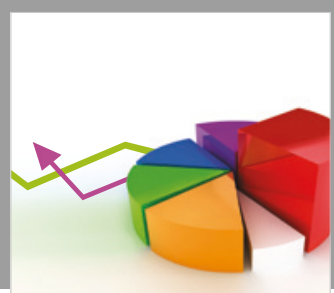

Journal of

Probability and Statistics
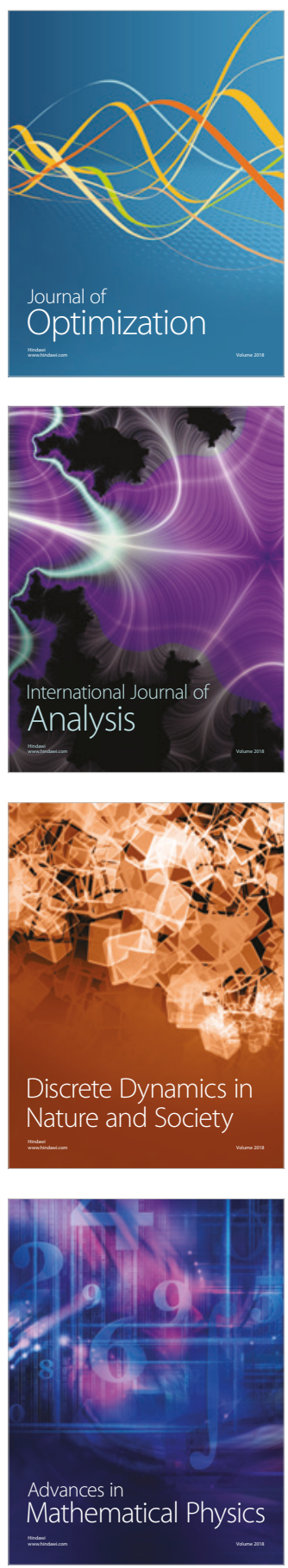